\title{
Toxic Animal-Based Medicinal Materials Can Be Effective in Treating Endometriosis: A Scoping Review
}

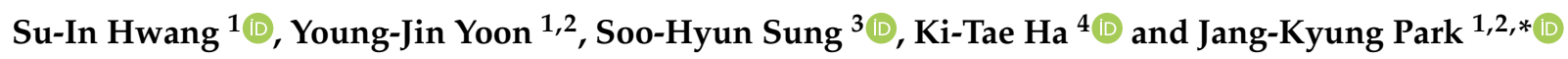 \\ 1 Department of Korean Medicine Obstetrics and Gynecology, Pusan National University Korean Medicine \\ Hospital, Yangsan 50612, Korea; hwangsi1216@gmail.com (S.-I.H.); yyj@pusan.ac.kr (Y.-J.Y.) \\ 2 Division of Clinical Medicine, School of Korean Medicine, Pusan National University, Yangsan 50612, Korea \\ 3 Department of Policy Development, National Development Institute of Korean Medicine, Seoul 04554, Korea; \\ koyote10010@nikom.or.kr \\ 4 Department of Korean Medical Science, School of Korean Medicine, Pusan National University, \\ Yangsan 50612, Korea; hagis@pusan.ac.kr \\ * Correspondence: vivat314@pusan.ac.kr; Tel.: +82-55-360-5978; Fax: +82-55-360-5890
}

\section{check for}

updates

Citation: Hwang, S.-I.; Yoon, Y.-J.; Sung, S.-H.; Ha, K.-T.; Park, J.-K. Toxic Animal-Based Medicinal Materials Can Be Effective in Treating Endometriosis: A Scoping Review. Toxins 2021, 13, 145. https://doi.org/ $10.3390 /$ toxins 13020145

Received: 28 December 2020

Accepted: 12 February 2021

Published: 14 February 2021

Publisher's Note: MDPI stays neutral with regard to jurisdictional claims in published maps and institutional affiliations.

Copyright: (c) 2021 by the authors. Licensee MDPI, Basel, Switzerland. This article is an open access article distributed under the terms and conditions of the Creative Commons Attribution (CC BY) license (https:// creativecommons.org/licenses/by/ $4.0 /)$.

\begin{abstract}
Animal toxins and venoms have recently been developed as cancer treatments possessing tumor cell growth-inhibitory, antiangiogenesis, and proapoptotic effects. Endometriosis is a common benign gynecological disorder in reproductive-age women, and no definite treatment for this disorder is without severe side effects. As endometriosis and malignant tumors share similar characteristics (progressive, invasive, estrogen-dependent growth, and recurrence), animal toxins and venoms are thought to be effective against endometriosis. The objective of this study was to outline studies using toxic animal-based medicinal materials (TMM) as endometriosis treatment and to explore its clinical applicability. Preclinical and clinical studies using TMM were searched for in four databases from inception to October 2020. A total of 20 studies of TMM on endometriosis were included. In eight clinical studies, herbal medicines containing TMM were effective in relieving symptoms of endometriosis, with no side effects. In twelve experimental studies, the main therapeutic mechanisms of TMM against endometriosis were proapoptotic, antiangiogenesis, estrogen levelreducing, and possible anti-inflammatory effects. TMM are thus considered promising sources for the development of an effective treatment method for endometriosis. Further studies are needed to clarify the therapeutic mechanism of TMM against endometriosis and to provide sufficient grounds for clinical application.
\end{abstract}

Keywords: endometriosis; toxic animal-based medicinal materials; Hirudo; Eupolyphaga; Scolopendra; Scorpio

Key Contribution: Toxic animal-based medicinal materials have traditionally been used in Chinese medicine for blood-activating and stasis-dispelling purposes. This study reviewed preclinical and clinical studies of toxic animal-based medicinal materials for endometriosis treatment.

\section{Introduction}

Endometriosis is a common estrogen-dependent, chronic inflammatory disease experienced by $10-15 \%$ of reproductive-age women [1-3]. It is defined as a disease in which endometrium-like tissue exists outside the uterus [2]. Endometriosis negatively affects the quality of life and well-being of women of childbearing age owing to distressing and debilitating symptoms and complications [4]. The most common clinical symptoms are dysmenorrhea, pelvic pain, infertility, and various clinical symptoms, such as dyspareunia, anal pain, and abdominal and adnexal masses $[5,6]$. Hypotheses on the origin of endometriosis occurrence include endometrial origin due to retrograde menstruation and non-endometrial origin, such as coelomic metaplasia and lymphatic and vascular metastasis [4,7]. The interaction between endocrine, immunologic, pro-inflammatory, and 
proangiogenic processes appears to be involved in the development of endometriosis [2]. In addition, endometriosis is a benign disease, but it displays similar features to malignancy, such as progressive, invasive, estrogen-dependent growth, and recurrence $[8,9]$.

Conventional treatments for endometriosis include analgesics, combined oral contraceptives, gonadotropin-releasing hormone agonists, and surgery $[4,10,11]$. However, there are limitations such as unpleasant and sometimes dangerous side effects and high recurrence rates [5]. Thus, patients with endometriosis seek traditional Chinese medicine treatments to cure the primary lesions and relieve symptoms, with fewer side effects [12-14]. In traditional Chinese medicine, "blood stasis" is considered the basic etiology of endometriosis, and thus treatment is aimed at removing the stasis by promoting blood circulation [15] In east Asian countries, animal-based medicinal materials, which eliminate blood stasis and facilitate blood circulation, have traditionally been used to treat diseases. In Korea, there are records that such materials were used to address gynecological diseases caused by blood stasis [16]. It seems that animal-based medicinal materials were used mainly in China, where such substances are widely in use to treat various diseases such as rheumatism [17], kidney disease [18], gynecological diseases [19], and cardiovascular and cerebrovascular diseases [20].

Venomous and poisonous animals have long been an area of interest in the treatment of diseases. Animals produce toxins in specialized glands or cells to protect themselves from predators and to predate on other animals [21]. Animal toxins include "poison", which is produced by specialized cells or tissues and transmitted via ingestion or contact, and "venom", which is produced in tissues or organs and delivered parenterally via specialized apparatus such as stingers, teeth, and nematocysts. [22]. Animal poisons and venoms are composed of different classes of molecules, and they contain abundant proteins, peptides, and neurotransmitters [21,22]. Although our understanding of the complexity of animal toxins is still limited, recent studies have shown that animal toxins can be developed as cancer treatments because of their ability to inhibit cell growth, inhibit angiogenesis, and induce apoptosis $[23,24]$.

Considering the pathophysiology of endometriosis and the treatment mechanism of animal poisons and venoms identified in previous studies, various animal poisons and venoms are thought to be effective against endometriosis. Recently, preclinical and clinical studies investigating the effects of animal poisons and venoms on endometriosis have been reported. However, as far as we know, no review articles on studies using toxic animalbased medicinal materials to treat endometriosis have been published. Therefore, in this study, we outline the use of toxic animal-based medicinal materials for endometriosis and explore the possibility of its clinical use.

\section{Materials and Methods}

\subsection{Scope of Toxic Animal-Based Medicinal Materials}

There are 99 animal-based medicinal materials with seven specifications (Korean Pharmacopoeia, Korean Herbal Pharmacopoeia, Chinese Pharmacopoeia, Japanese Pharmacopoeia, Japanese standards for non-Pharmacopoeial crude drugs, Taiwan Herbal Pharmacopoeia, Democratic People's Republic of Korea Pharmacopoeia) from five northeast Asian countries [25]. Among them, the following 10 toxic animal-based medicinal materials were selected by identifying toxicity [26]: Vespae Nidus, Tabanus, Mylabris, Agkistrodon, Bufonis Venenum, Hirudo, Scolopendra, Eupolyphaga, Scorpio, and Gecko.

\subsection{Literature Search Strategy}

We searched the following databases from inception to October 2020: MEDLINE, EMBASE, one Chinese database (China National Knowledge Infrastructure (CNKI)), and one Korean database (Oriental Medicine Advanced Searching Integrated System (OASIS)). The search words were "Vespae Nidus OR Nidus Vespae OR Bee venom OR Tabanus OR Mylabris OR Cantharides OR Agkistrodon OR Snake venom OR Bufonis Venenum OR Bufo OR Bufo Siccus OR Toad venom OR Bufonis Crustum OR Toad cake OR Secretio 
Bufonis OR Hirudo OR Leech OR Scolopendra OR Scolopendrae OR Eupolyphaga OR Steleophaga OR Scorpio OR Gecko OR Tokay" AND "endometriosis". There were no language limitations in this study.

\subsection{Inclusion and Exclusion Criteria}

Type of studies: Experimental studies, such as animal or cell studies, and clinical studies using toxic animal-based medicinal materials for treating endometriosis were included in this review. All types of clinical studies, including randomized controlled trials (RCTs), observational studies, cohort studies, case reports, and case series were included in this review. Literature studies, qualitative studies, and surveys were excluded.

Subjects: Clinical studies with female patients with endometriosis were included. Experimental studies on animal endometriosis models or cell culture studies were considered for inclusion.

Types of intervention: Studies using 10 types of toxic animal-based medicinal materials in the treatment of endometriosis patients, endometriosis animal models, and endometriosis cells were included. In the case of toxic animal-based medicinal materials alone, a combination of toxic animal-based medicinal materials and other toxic medicinal materials was also included.

\subsection{Study Selection and Data Extraction}

Two independent authors (SIH and SHS) selected the studies according to the predefined criteria. Any disagreements were discussed by a third author (JKP). Two authors (SIH, GTH) independently extracted the following data from the selected experimental studies: author, year, study design, toxic animal-based medicinal materials used in intervention, target cell (in vitro)/animal model (in vivo), dosage, treatment period, outcome measures, results, and components of herbal medicine. Two authors (SHS, YJY) independently extracted the following data from clinical studies: author, study design, sample size, age, main symptoms, toxic animal-based medicinal materials used in intervention, control intervention, treatment duration, outcome measures, main results, adverse events, components of herbal medicine, dosage, frequency, and dosing period. We resolved any disagreements by discussing with a third author (JKP).

\section{Results}

After literature searching, we identified a total of 153 studies (6 in English database, 146 in Chinese database, and 1 in Korean database), from which 74 duplicate studies were excluded. By screening the titles and abstracts of 79 studies, 11 studies were found to be irrelevant due to the study type (10 reviews and 1 survey), 25 studies irrelevant due to the subject, and 13 studies irrelevant due to the intervention were excluded. After assessing the full text of the remaining 30 studies for eligibility, 20 studies were finally included, excluding one study due to irrelevance in the study type, one study due to irrelevance in the subject, three studies due to irrelevance in the intervention, and five studies due to insufficient details about the composition of the herbal medicine. Of the 20 studies finally selected, 8 were experimental studies and 12 were clinical studies. A flow diagram of the study selection process is shown in Figure 1. 

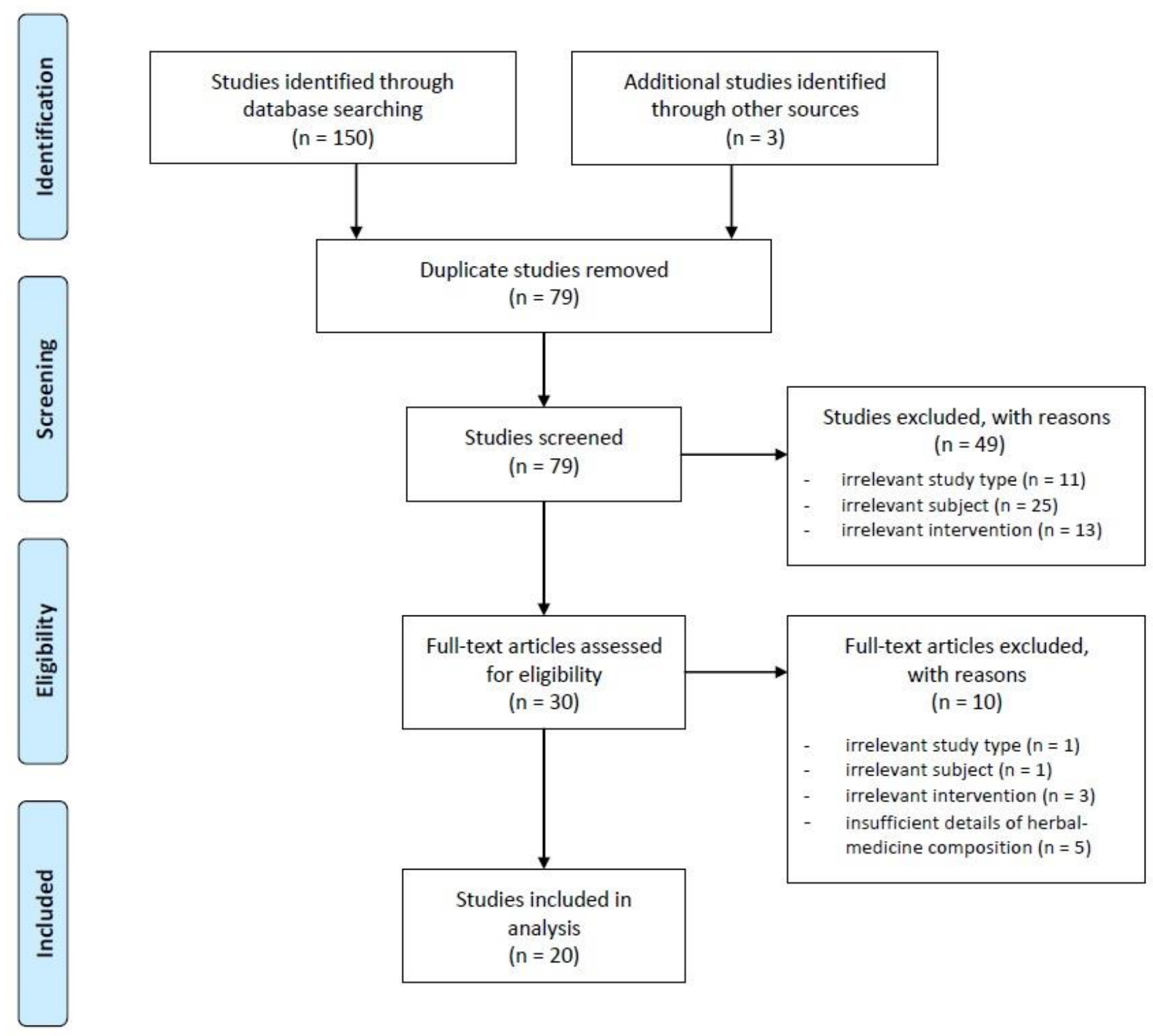

Figure 1. Flow chart of the study selection process. After literature searching, we identified a total of 153 studies, from which 74 duplicate studies were excluded. We screened 79 studies' titles and abstracts. As a result, 49 studies were excluded, and 30 studies passed the first round of the selection process. Of these 30 studies, 10 irrelevant studies were excluded again, and the final 20 studies were selected for this study. Of the 20 studies finally selected, 8 were experimental studies and 12 were clinical studies.

\subsection{Preclinical Studies}

Among the studies included in this analysis, there were eight preclinical studies [27-34] investigating the effect of animal toxins on endometriosis, two in vitro studies $[28,30]$, one both in vivo and in vitro study [29], and five in vivo studies [27,31-34].

The medicinal interventions used in the eight studies were largely divided into three categories, namely medicinal compounds extracted from animal toxins in two studies $[28,29]$, individual medicinal extracts in two studies $[27,34]$, and medicinal decoctions in four studies [30-33]. The composition of the medicinal formulae used in the four studies is summarized in Table 1. 
Table 1. Composition of the herbal medicines used in the included preclinical studies.

\begin{tabular}{|c|c|c|}
\hline $\begin{array}{l}\text { First Author } \\
\text { (Year) }\end{array}$ & Herbal Medicine & Component \\
\hline $\begin{array}{c}\text { Liu } \\
\text { (2014) [30] }\end{array}$ & Pingchongjiangnifang & $\begin{array}{l}\text { Cinnamomi Ramulus } 15 \mathrm{~g} \text {, Draconis Sanguis } 3 \text { g, Trogopterorum Faeces } \\
10 \text { g, Typhae Pollen } 6 \text { g, Aquilariae Lignum } 6 \text { g, Hirudo } 3 \text { g, Liriopis seu } \\
\text { Ophiopogonis Tuber } 10 \text { g, Glycyrrhizae Radix et Rhizoma } 6 \text { g }\end{array}$ \\
\hline $\begin{array}{c}\mathrm{Xu} \\
(2018)[31]\end{array}$ & Eleng Capsule & $\begin{array}{c}\text { Curcumae Rhizoma } 10 \text { g, Sparganii Rhizoma } 10 \text { g, Salviae Miltiorrhizae } \\
\text { Radix } 15 \text { g, Curcumae Radix } 15 \text { g, Paeoniae Radix Rubra } 15 \text { g, Pelodiscis } \\
\text { Carapax } 15 \text { g, Hirudo } 3 \text { g }\end{array}$ \\
\hline $\begin{array}{c}\text { Liu } \\
\text { (2020) [32] }\end{array}$ & Huangzhi Neiyi Capsule & Hirudo 3 g, Rhei Radix et Rhizoma 9 g, Cyperi Rhizoma 6 g \\
\hline $\begin{array}{c}\text { Wang } \\
\text { (2005) [33] }\end{array}$ & Wudan Wan Modified Decoction & $\begin{array}{l}\text { Salviae Miltiorrhizae Radix } 10 \text { g, Paeoniae Radix Rubra } 10 \text { g, Curcumae } \\
\text { Rhizoma } 10 \text { g, Cinnamomi Cortex } 6 \text { g, Corydalis Tuber } 10 \text { g, Hirudo } 10 \text { g, } \\
\text { Scolopendra two pieces, Pelodiscis Carapax } 15 \text { g, Sappan Lignum } 10 \mathrm{~g}\end{array}$ \\
\hline
\end{tabular}

Among the toxic animal-based medical materials, Hirudo was the most commonly used in medicinal decoctions [30-33], followed by bufalin twice [28,29].

According to the eight studies, the underlying mechanisms of animal toxins against endometriosis were as follows: induction of apoptosis [28-30], inhibition of angiogenesis [31,32], reduction of estrogen level [27], suppression of matrix metalloproteinase (MMP)-9 expression [34], and anti-inflammatory effect [33] (Table 2).

\subsection{Clinical Studies}

In this analysis, we included a total of 12 clinical studies [35-46] examining the therapeutic effects of herbal medicine containing animal toxins on endometriosis, of which five were case series [35-39] and seven were randomized controlled trials (RCTs) [40-46]. According to these studies, dysmenorrhea was the most common symptom in patients diagnosed with endometriosis, followed by infertility, pelvic mass, dyspareunia, pelvic pain, mittelschmerz, and anal pain.

In all the clinical studies, medicinal decoctions were used. Quyu Jiedu Xiaozheng Decoction was used in two studies, but at different doses [38,41]. Other than that, different prescriptions were used in the remaining studies. The toxic animal-based medicinal materials used in these studies include Hirudo, Scolopendra, Eupolyphaga, and Scorpio. Hirudo was used in a total of eight studies [35-37,39,40,43,44,46] at daily doses of $0.5 \mathrm{~g}$ [37], 1-2 g [43], 4-5 g [40], $6 \mathrm{~g}$ [39], $9 \mathrm{~g}$ [35], and $10 \mathrm{~g}$ [44,46]. Scolopendra was used in five studies $[37,38,41,45,46]$ at varying doses of $0.5 \mathrm{~g}$ [37], one to two pieces [36], two pieces [41,46], and $6 \mathrm{~g}$ [45] per day. Eupolyphaga was used in four studies [35,38,41,46] at doses of $6 \mathrm{~g}$ [38,41], $10 \mathrm{~g}$ [46], and $12 \mathrm{~g}$ [35] per day. Scorpio was used in one study [42] at a dose of $6 \mathrm{~g}$ per day. The administration duration varied from 1 to 9 months, with 3 months [36-38,44-46] being the most common. The timing of administration also varied; some decoctions were discontinued during the menstrual period [35,39], administered throughout the menstrual cycle $[40,42,44,45]$, or administered after menstruation $[37,46]$.

All included clinical studies reported improvement of symptoms related to endometriosis, and three studies $[37,42,45]$ reported that no side effects were observed (Table 3$)$. The dosing regimens of herbal medicines containing toxic animal-based medicinal materials in the included clinical studies are presented in Table 4. 
Table 2. Characteristics of the included preclinical studies.

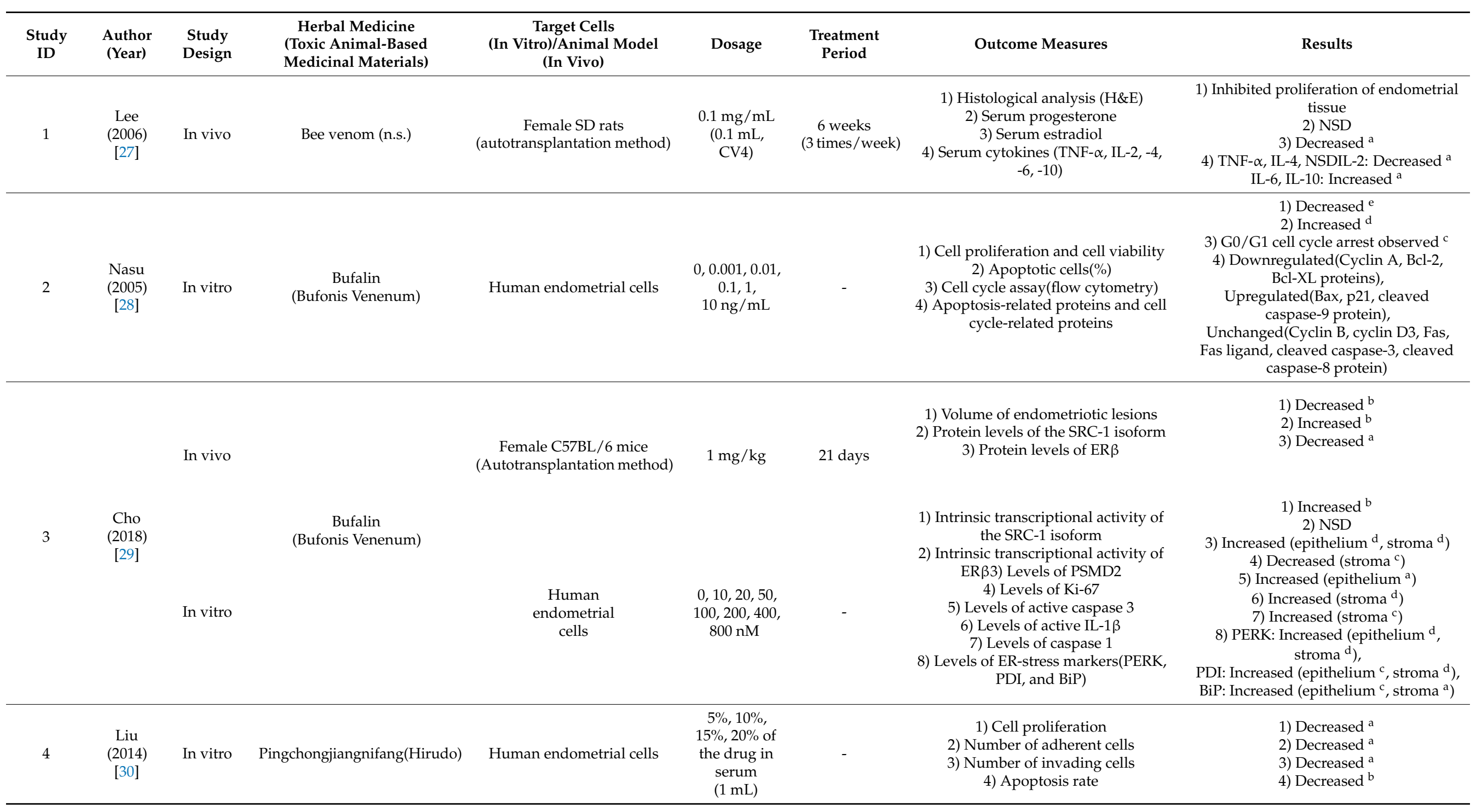


Table 2. Cont.

\begin{tabular}{|c|c|c|c|c|c|c|c|c|}
\hline $\begin{array}{l}\text { Study } \\
\text { ID }\end{array}$ & $\begin{array}{l}\text { Author } \\
\text { (Year) }\end{array}$ & $\begin{array}{l}\text { Study } \\
\text { Design }\end{array}$ & $\begin{array}{c}\text { Herbal Medicine } \\
\text { (Toxic Animal-Based } \\
\text { Medicinal Materials) }\end{array}$ & $\begin{array}{c}\text { Target Cells } \\
\text { (In Vitro)/Animal Model } \\
\text { (In Vivo) }\end{array}$ & Dosage & $\begin{array}{l}\text { Treatment } \\
\text { Period }\end{array}$ & Outcome Measures & Results \\
\hline 5 & $\begin{array}{c}\mathrm{Xu} \\
(2018) \\
{[31]}\end{array}$ & In vivo & Eleng Capsule(Hirudo) & $\begin{array}{c}\text { Female SD rats } \\
\text { (autotransplantation method) }\end{array}$ & $\begin{array}{c}4 \mathrm{~g} / \mathrm{kg} \\
\text { (high dose), } \\
2 \mathrm{~g} / \mathrm{kg} \\
\text { (medium } \\
\text { dose), } \\
1 \mathrm{~g} / \mathrm{kg} \\
\text { (low dose) }\end{array}$ & 28 days & $\begin{array}{l}\text { 1) Volume of endometriotic foci } \\
\text { 2) Histological analysis (H\&E) } \\
\text { 3) Serum levels of VEGF } \\
\text { 4) Serum levels of bFGF } \\
\text { 5) Serum levels of PDGF } \\
\text { 6) Expression of VEGF } \\
\text { 7) Expression of bFGF } \\
\text { 8) Expression of PDGF } \\
\text { 9) Expression of NF-kB } \\
\text { 10) Expression of TLR4 }\end{array}$ & 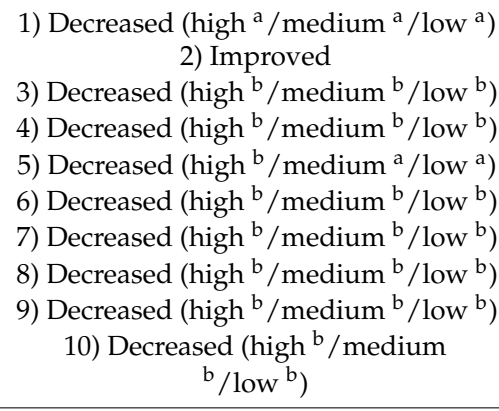 \\
\hline 6 & $\begin{array}{l}\text { Liu } \\
(2020) \\
{[32]}\end{array}$ & In vivo & $\begin{array}{l}\text { Huangzhi Neiyi Capsule } \\
\text { (Hirudo) }\end{array}$ & $\begin{array}{c}\text { Female SD rats } \\
\text { (autotransplantation method) }\end{array}$ & $\begin{array}{c}9 \mathrm{~g} / \mathrm{kg} \\
\text { (high dose), } \\
4.5 \mathrm{~g} / \mathrm{kg} \\
\text { (low dose) }\end{array}$ & 28 days & $\begin{array}{l}\text { 1) PCNA } \\
\text { 2) CD31 } \\
\text { 3) VEGF in peritoneal fluid } \\
\text { 4) mRNA expression of VEGF } \\
\text { 5) mRNA expression of HIF-1 } \alpha\end{array}$ & 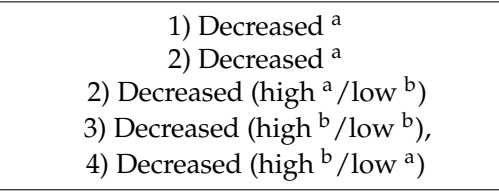 \\
\hline 7 & $\begin{array}{l}\text { Wang } \\
(2005) \\
{[33]}\end{array}$ & In vivo & $\begin{array}{l}\text { Wudan Wan Modified } \\
\text { Decoction } \\
\text { (Hirudo, Scolopendra) }\end{array}$ & $\begin{array}{c}\text { Female SD rats } \\
\text { (autotransplantation method) }\end{array}$ & $\begin{array}{c}1 \mathrm{~mL} / 100 \mathrm{~g} \\
(26.56 \mathrm{~g} / \mathrm{kg}) \\
\text { (high dose), } \\
1 / 2 \text { diluted } \\
\text { (medium } \\
\text { dose), } \\
1 / 4 \text { diluted } \\
\text { (low dose) }\end{array}$ & 28 days & $\begin{array}{l}\text { 1) TER for disappearance of ectopic } \\
\text { cysts } \\
\text { 2) Effects on abdominal inflammation } \\
\text { 3) TER for ectopic endometrial atrophy }\end{array}$ & $\begin{array}{l}\left.\text { 1) Improved (medium }{ }^{\text {a }} / \text { high }^{a}\right), \text { NSD } \\
\text { (low) } \\
\text { 2) Less severe adhesion (medium/high), } \\
\text { More severe adhesion (low) } \\
\text { 3) Improved (medium }{ }^{b} / \text { high }^{\mathrm{a}} \text { ), NSD } \\
\text { (low) }\end{array}$ \\
\hline \multirow[t]{2}{*}{8} & \multirow[t]{2}{*}{$\begin{array}{c}\text { Zhang } \\
(2015) \\
{[34]}\end{array}$} & In vivo & \multirow[t]{2}{*}{$\begin{array}{l}\text { Gloydius brevicaudus venom } \\
\text { (Snake venom) }\end{array}$} & $\begin{array}{c}\text { Female SD rats } \\
\text { (autotransplantation method) }\end{array}$ & $\begin{array}{l}0.75 \mathrm{mg} / \mathrm{kg} \\
\text { (low dose), } \\
1.5 \mathrm{mg} / \mathrm{kg} \\
\text { (high dose) }\end{array}$ & 3 weeks & $\begin{array}{l}\text { 1) Volume of ectopic foci } \\
\text { 2) Expression of MMP-9 }\end{array}$ & $\begin{array}{l}\text { 1) Decreased a } \\
\text { 2) Decreased }\end{array}$ \\
\hline & & In vitro & & Rat ectopic endometrial cells & $\begin{array}{l}\text { Disintegrin } \\
1.5 \mu \mathrm{g} / \mathrm{mL}\end{array}$ & - & 1) Expression of MMP-9 & 1) Decreased ${ }^{b}$ \\
\hline
\end{tabular}

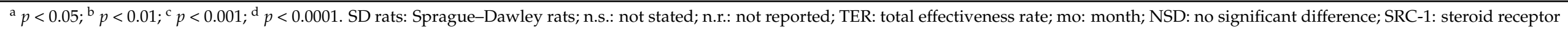

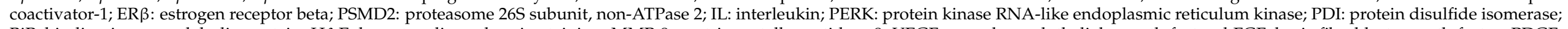

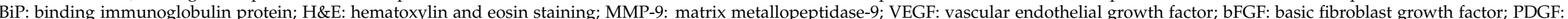

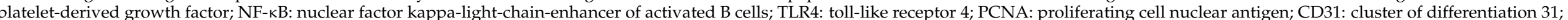

HIF-1 $\alpha$ : hypoxia-inducible factor 1-alpha. 
Table 3. Characteristics of the included clinical studies.

\begin{tabular}{|c|c|c|c|c|c|c|c|c|c|c|c|c|}
\hline $\begin{array}{l}\text { Study } \\
\text { ID }\end{array}$ & $\begin{array}{l}\text { Author } \\
\text { (Year) }\end{array}$ & $\begin{array}{l}\text { Study } \\
\text { Design }\end{array}$ & $\begin{array}{c}\text { Sample } \\
\text { Size } \\
\text { (TG/CG) }\end{array}$ & $\begin{array}{c}\text { Mean } \\
\text { Age } \pm \text { SD } \\
\text { (Min, Max) }\end{array}$ & Main Symptoms & Diagnostic Criteria & Intervention & $\begin{array}{c}\text { Control } \\
\text { Intervention }\end{array}$ & $\begin{array}{c}\text { Treatment } \\
\text { Period }\end{array}$ & Outcome Measures & Main Results & $\mathrm{AE}$ \\
\hline 1 & $\begin{array}{c}\text { Wu } \\
(1993) \\
{[35]}\end{array}$ & $\begin{array}{l}\text { Case } \\
\text { series }\end{array}$ & 60 & $\begin{array}{c}\text { n.r. } \\
(20,48)\end{array}$ & $\begin{array}{l}\text { Dysmenorrhea, } \\
\text { infertility, } \\
\text { menstrual disorder }\end{array}$ & $\begin{array}{l}\text { Symptom, } \\
\text { gynecological exam, } \\
\text { ultrasonography }\end{array}$ & $\begin{array}{l}\text { Herbal } \\
\text { medicine }\end{array}$ & - & n.r. & 1) TER & $91.67 \%$ & n.r. \\
\hline 2 & $\begin{array}{l}\text { Zhang } \\
(2007) \\
{[36]}\end{array}$ & $\begin{array}{l}\text { Case } \\
\text { series }\end{array}$ & 30 & $\begin{array}{c}32 \\
(23,43)\end{array}$ & $\begin{array}{l}\text { Dysmenorrhea, } \\
\text { mittelschmerz, } \\
\text { endometrioma }\end{array}$ & $\begin{array}{c}\text { Symptom, } \\
\text { gynecological exam, } \\
\text { ultrasonography }\end{array}$ & $\begin{array}{l}\text { Herbal } \\
\text { medicine }\end{array}$ & - & $3 \mathrm{mo}$ & VAS & Positive $^{\mathrm{a}}$ & n.r. \\
\hline 4 & $\begin{array}{l}\text { Zhang } \\
(2007) \\
{[38]}\end{array}$ & $\begin{array}{l}\text { Case } \\
\text { series }\end{array}$ & 78 & $\begin{array}{c}34.5 \\
(21,46)\end{array}$ & $\begin{array}{l}\text { Dysmenorrhea, } \\
\text { pelvic mass, } \\
\text { infertility }\end{array}$ & $\begin{array}{l}\text { Gynecological exam, } \\
\text { ultrasonography, } \\
\text { laparoscopy }\end{array}$ & $\begin{array}{l}\text { Herbal } \\
\text { medicine }\end{array}$ & - & $3 \mathrm{mo}$ & $\begin{array}{l}\text { 1) TER for } \\
\text { dysmenorrhea } \\
\text { 2) TER for pelvic } \\
\text { mass } \\
\text { 3) Pregnancy rate } \\
\text { (within 2 years) } \\
\end{array}$ & $\begin{array}{l}\text { 1) } 94.11 \% \\
\text { 2) } 87.5 \% \\
\text { 3) } 19.05 \%\end{array}$ & n.r. \\
\hline 6 & $\begin{array}{l}\text { Han } \\
(2009) \\
{[40]}\end{array}$ & RCT & $\begin{array}{c}156 \\
(78 / 78)\end{array}$ & $\begin{array}{c}28.5 \\
(18,48)\end{array}$ & n.r. & $\begin{array}{l}\text { Symptom, } \\
\text { laparoscopy }\end{array}$ & $\begin{array}{c}\text { Herbal } \\
\text { medicine(A) }\end{array}$ & $\begin{array}{c}\text { Herbal } \\
\text { medicine(B) }\end{array}$ & $9 \mathrm{mo}$ & $\begin{array}{l}\text { 1) TER } \\
\text { 2) Pregnancy rate } \\
\text { 3) Recurrence rate }\end{array}$ & $\begin{array}{l}\text { 1) Positive }{ }^{b} \\
\text { 2) } 11.53 \%(\text { TG }) \text { vs } \\
0 \%(C G) \\
\text { 3) Positive }{ }^{b}\end{array}$ & n.r. \\
\hline 7 & $\begin{array}{c}\text { Zhang } \\
(2009) \\
{[41]}\end{array}$ & RCT & $\begin{array}{c}128 \\
(45 / 43 / 40)\end{array}$ & $\begin{array}{c}\text { n.r. } \\
\text { (n.r.) }\end{array}$ & $\begin{array}{l}\text { Dysmenorrhea, } \\
\text { pelvic mass }\end{array}$ & $\begin{array}{c}\text { Symptom, } \\
\text { gynecological exam, } \\
\text { ultrasonography, } \\
\text { laparoscopy, } \\
\text { antiendometrial } \\
\text { antibody, CA125 }\end{array}$ & $\begin{array}{c}\text { Herbal } \\
\text { medicine(A) }\end{array}$ & $\begin{array}{l}\text { CG1: Herbal } \\
\text { medicine(B) } \\
\text { CG2: Herbal } \\
\text { medicine(C) }\end{array}$ & $3-6 \mathrm{mo}$ & $\begin{array}{l}\text { 1) TER } \\
\text { 2) Dysmenorrhea } \\
\text { score } \\
\text { 3) Pelvic mass score }\end{array}$ & $\begin{array}{l}\text { 1) Positive (vs CG1 a } \\
\text { /CG2 }{ }^{a} \text { ) } \\
\text { 2) Positive (vs CG1 }{ }^{b} \\
\left./ C G 2^{b}\right) \\
\text { 3) Positive (vs CG1 }{ }^{b} \\
\left./ C G 2^{b}\right)\end{array}$ & n.r. \\
\hline 8 & $\begin{array}{l}\text { Lin } \\
(2011) \\
{[42]}\end{array}$ & $\mathrm{RCT}$ & $\begin{array}{c}70 \\
(40 / 30)\end{array}$ & $\begin{array}{c}\text { n.r. } \\
\text { (n.r.) }\end{array}$ & Dysmenorrhea & $\begin{array}{l}\text { Symptom, } \\
\text { ultrasonography, } \\
\text { CA125 }\end{array}$ & $\begin{array}{l}\text { Herbal } \\
\text { medicine }\end{array}$ & Mifepristone & $6 \mathrm{mo}$ & $\begin{array}{l}\text { 1) TER } \\
\text { 2) TER for } \\
\text { dysmenorrhea } \\
\text { 3) CA125 }\end{array}$ & $\begin{array}{l}\text { 1) NSD } \\
\text { 2) Positive a } \\
\text { 3) Positive }{ }^{a}\end{array}$ & none \\
\hline
\end{tabular}


Table 3. Cont.

\begin{tabular}{|c|c|c|c|c|c|c|c|c|c|c|c|c|}
\hline $\begin{array}{l}\text { Study } \\
\text { ID }\end{array}$ & $\begin{array}{l}\text { Author } \\
\text { (Year) }\end{array}$ & $\begin{array}{l}\text { Study } \\
\text { Design }\end{array}$ & $\begin{array}{l}\text { Sample } \\
\text { Size } \\
\text { (TG/CG) }\end{array}$ & $\begin{array}{c}\text { Mean } \\
\text { Age } \pm \text { SD } \\
\text { (Min, Max) }\end{array}$ & Main Symptoms & Diagnostic Criteria & Intervention & $\begin{array}{c}\text { Control } \\
\text { Intervention }\end{array}$ & $\begin{array}{c}\text { Treatment } \\
\text { Period }\end{array}$ & Outcome Measures & Main Results & $\mathrm{AE}$ \\
\hline 9 & $\begin{array}{c}\text { Meng } \\
(2012) \\
{[43]}\end{array}$ & $\mathrm{RCT}$ & $\begin{array}{c}312 \\
(156 / 156)\end{array}$ & $\begin{array}{c}28.5 \\
(18,48)\end{array}$ & n.r. & $\begin{array}{l}\text { Symptom, } \\
\text { gynecological exam, } \\
\text { ultrasonography }\end{array}$ & $\begin{array}{l}\text { Herbal } \\
\text { medicine(A) } \\
+ \text { Control } \\
\text { intervention }\end{array}$ & $\begin{array}{c}\text { Herbal } \\
\text { medicine(B) }\end{array}$ & $9 \mathrm{mo}$ & $\begin{array}{l}\text { 1) TER } \\
\text { 2) Pregnancy rate } \\
\text { 3) Recurrence rate }\end{array}$ & $\begin{array}{l}\text { 1) Positive }{ }^{\mathrm{b}} \\
\text { 2) } 11.54 \%(\mathrm{TG}) \mathrm{vs} \\
0 \%(\mathrm{CG}) \\
\text { 3) Positive } \\
\text { b }\end{array}$ & n.r. \\
\hline 10 & $\begin{array}{l}\text { Guo } \\
(2013) \\
{[44]}\end{array}$ & $\mathrm{RCT}$ & $\begin{array}{c}94 \\
(48 / 46)\end{array}$ & $\begin{array}{c}\text { TG: } 32.0 \pm 9.6 \\
\quad(22,42) \\
\text { CG: } 31.0 \pm 7.8 \\
(23,40)\end{array}$ & Infertility & $\begin{array}{c}\text { Symptom, } \\
\text { gynecological exam, } \\
\text { ultrasonograph, } \\
\text { CA125 }\end{array}$ & $\begin{array}{c}\text { Herbal } \\
\text { medicine } \\
+ \text { Control } \\
\text { intervention }\end{array}$ & $\begin{array}{l}\text { Desogestrel } \\
\text { and Ethinyl } \\
\text { Estradiol }\end{array}$ & $3 \mathrm{mo}$ & Pregnancy rate & Positive ${ }^{b}$ & n.r. \\
\hline 11 & $\begin{array}{c}\text { Liu } \\
(2017) \\
{[45]}\end{array}$ & $\mathrm{RCT}$ & $\begin{array}{c}72 \\
(36 / 36)\end{array}$ & $\begin{array}{c}\text { n.r. } \\
(24,45)\end{array}$ & Dysmenorrhea & $\begin{array}{l}\text { Symptom, } \\
\text { gynecological exam, } \\
\text { ultrasonography }\end{array}$ & $\begin{array}{l}\text { Herbal } \\
\text { medicine }\end{array}$ & $\begin{array}{l}\text { Sanjie } \\
\text { Zhentong } \\
\text { Capsule }\end{array}$ & $3 \mathrm{mo}$ & $\begin{array}{l}\text { 1) TER } \\
\text { 2) VAS }\end{array}$ & $\begin{array}{l}\text { 1) Positive }{ }^{a} \\
\text { 2) } \text { Positive }^{a}\end{array}$ & none \\
\hline 12 & $\begin{array}{c}\mathrm{Yi} \\
(2018) \\
{[46]}\end{array}$ & $\mathrm{RCT}$ & $\begin{array}{c}60 \\
(30 / 30)\end{array}$ & $\begin{array}{c}\text { TG: } \\
\text { 29.41 } 44.7 \\
\text { (n.r.) } \\
\text { CG: } \\
30.0 \pm 4.1 \text { (n.r.) }\end{array}$ & $\begin{array}{l}\text { Dysmenorrhea, } \\
\text { dyspareunia }\end{array}$ & $\begin{array}{c}\text { Symptom, } \\
\text { ultrasonography, } \\
\text { antiendometrial } \\
\text { antibody, CA125 }\end{array}$ & $\begin{array}{c}\text { Herbal } \\
\text { medicine } \\
+ \text { +Control } \\
\text { intervention }\end{array}$ & Gestrinone & $3 \mathrm{mo}$ & $\begin{array}{l}\text { 1) TER } \\
\text { 2) VAS }\end{array}$ & $\begin{array}{l}\text { 1) Positive }{ }^{a} \\
\text { 2) Positive }{ }^{a}\end{array}$ & n.r. \\
\hline
\end{tabular}

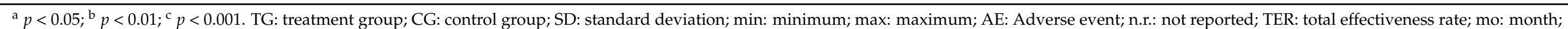

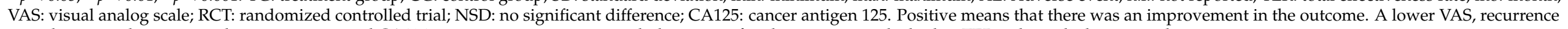
rate, dysmenorrhea score, pelvic mass score, and CA124 meant an improvement with the status of endometriosis, and a higher TER indicated a better condition.

Table 4. Dosing regimens of herbal medicines containing toxic animal-based medicinal materials in the included clinical studies.

\begin{tabular}{|c|c|c|c|c|c|c|}
\hline Study ID & $\begin{array}{c}\text { Author } \\
\text { (Year) }\end{array}$ & Herbal Medicine & Component & $\begin{array}{l}\text { Toxic Animal-Based } \\
\text { Medicinal Materials }\end{array}$ & Dosage, Frequency & Dosing Period \\
\hline 1 & $\begin{array}{c}\text { Wu } \\
(1993) \\
{[35]}\end{array}$ & n.r. & $\begin{array}{l}\text { Sparganii Rhizoma } 9 \text { g, Curcumae Rhizoma } 8 \text { g, } \\
\text { Liquidambaris Fructus } 9 \text { g, Hirudo } 9 \text { g, Paeoniae Radix } \\
\text { Rubra } 9 \text { g, Manitis Squama } 12 \mathrm{~g} \text {, Eupolyphaga } 12 \mathrm{~g} \text {, } \\
\text { Moutan Radicis Cortex } 12 \mathrm{~g} \text {, Salviae Miltiorrhizae Radix } \\
12 \mathrm{~g} \text {, Cyperi Rhizoma } 12 \mathrm{~g} \text {, Prunellae Spica } 12 \mathrm{~g}\end{array}$ & Eupolyphaga, Hirudo & n.r. & $\begin{array}{l}\text { Medication } \\
\text { discontinued during } \\
\text { menstruation }\end{array}$ \\
\hline 2 & $\begin{array}{c}\text { Zhang } \\
(2007) \\
{[36]}\end{array}$ & n.r. & $\begin{array}{l}\text { Salviae Miltiorrhizae Radix, Persicae Semen, Corydalis } \\
\text { Tuber, Curcumae Rhizoma, Hirudo, Linderae Radix, } \\
\text { Olibanum, Myrrha, Cinnamomi Cortex }\end{array}$ & Hirudo & One dose per day, bid & n.r. \\
\hline
\end{tabular}


Table 4. Cont.

\begin{tabular}{|c|c|c|c|c|c|c|}
\hline Study ID & $\begin{array}{c}\text { Author } \\
\text { (Year) }\end{array}$ & Herbal Medicine & Component & $\begin{array}{l}\text { Toxic Animal-Based } \\
\text { Medicinal Materials }\end{array}$ & Dosage, Frequency & Dosing Period \\
\hline 3 & $\begin{array}{c}\mathrm{Xu} \\
(2007) \\
{[37]}\end{array}$ & $\begin{array}{l}\text { Leech Tongluo Capsule } \\
\text { (Shuizhi tongluo jiaonang) }\end{array}$ & $\begin{array}{c}\text { Hirudo } 0.5 \mathrm{~g} \text {, Manitis Squama } 0.5 \mathrm{~g} \text {, Astragali Radix } 0.5 \mathrm{~g} \text {, } \\
\text { Draconis Sanguis } 0.5 \mathrm{~g} \text {, Codonopsis Pilosulae Radix } 0.5 \mathrm{~g} \text {, } \\
\text { Sparganii Rhizoma 0.5 g, Curcumae Rhizoma 0.5 g, } \\
\text { Scolopendra 0.5 g, Cnidii Rhizoma 0.5 g, Glycyrrhizae } \\
\text { Radix et Rhizoma } 0.5 \mathrm{~g}\end{array}$ & Hirudo, Scolopendra & $\begin{array}{c}\text { Six granules/time, } \\
\text { tid }\end{array}$ & From MCD 5 \\
\hline 4 & $\begin{array}{c}\text { Zhang } \\
(2007) \\
{[38]}\end{array}$ & $\begin{array}{l}\text { Quyu Jiedu Xiaozheng } \\
\text { Decoction } \\
\text { (Quyu Jiedu Xiaozheng tang) }\end{array}$ & $\begin{array}{l}\text { Sargentodoxa cuneata } 15-30 \text { g, Hedyotidis Herba } 15-30 \text { g, } \\
\text { Astragali Radix } 30 \text { g, Coicis Semen } 30 \text { g, Scapharcae seu } \\
\text { Tegillarcae Concha } 15 \text { g, Litchi Semen } 15 \text { g, Cremastrae } \\
\text { Tuber 9-12 g, Draconis Sanguis 6-9 g, Olibanum 6-9 g, } \\
\text { Myrrha 6-9 g, Eupolyphaga } 6 \text { g, Scolopendra 1-2 pieces, } \\
\text { Agastachis Herba } 9 \text { g, Glycyrrhizae Radix et Rhizoma } 6 \text { g }\end{array}$ & Eupolyphaga, Scolopendra & One dose per day, n.r. & n.r. \\
\hline 5 & $\begin{array}{c}\text { Feng } \\
(2014) \\
{[39]}\end{array}$ & Xiaoyi Zhuyun tang & $\begin{array}{c}\text { Angelicae Gigantis Radix } 12 \text { g, Paeoniae Radix Rubra } \\
12 \text { g, Paeoniae Radix Alba } 12 \text { g, Cyperi Rhizoma } 12 \text { g, } \\
\text { Morindae Radix } 12 \text { g, Patriniae Radix } 12 \text { g, Sparganii } \\
\text { Rhizoma } 20 \text { g, Curcumae Rhizoma } 20 \text { g, Astragali Radix } \\
20 \text { g, Galli Gigeriae Endothelium Corneum } 20 \text { g, } \\
\text { Gleditsiae Spina } 20 \text { g, Lycopi Herba } 20 \text { g, Dipsaci Radix } \\
20 \text { g, Drynariae Rhizoma } 20 \text { g, Eucommiae Cortex } 20 \text { g, } \\
\text { Coicis Semen } 20 \text { g, Bupleuri Radix } 9 \text { g, Hirudo } 6 \text { g, Cervi } \\
\text { Cornu } 10 \text { g }\end{array}$ & Hirudo & One dose per day, bid & $\begin{array}{l}\text { From MCD } 6 \text { to MCD } \\
15 \text { (10 days) }\end{array}$ \\
\hline 6 & $\begin{array}{l}\text { Han } \\
(2009) \\
{[40]}\end{array}$ & $\begin{array}{ll}\text { A } & \text { n.r. } \\
\text { B } & \text { n.r. }\end{array}$ & $\begin{array}{c}\text { Hirudo } 4-5 \mathrm{~g} \\
\text { Salviae Miltiorrhizae Radix } 10-15 \mathrm{~g} \text {, Angelicae Gigantis } \\
\text { Radix 8-10 g, Salvia chinensis Benth 8-10 g, Myrrha } 10 \mathrm{~g}, \\
\text { Sparganii Rhizoma } 8 \text { g, Leonuri Herba } 8 \mathrm{~g}\end{array}$ & Hirudo & n.r., bid & $\begin{array}{l}\text { From } 3-4 \text { days before } \\
\text { menstruation to the end } \\
\text { of menstruation }\end{array}$ \\
\hline 7 & $\begin{array}{c}\text { Zhang } \\
(2009) \\
{[41]}\end{array}$ & $\begin{array}{c}\text { Quyu Jiedu Xiaozheng } \\
\text { Decoction } \\
\text { (Quyu Jiedu Xiaozheng tang) }\end{array}$ & $\begin{array}{l}\text { Sargentodoxa cuneata } 30 \mathrm{~g} \text {, Hedyotidis Herba } 30 \mathrm{~g} \text {, Coicis } \\
\text { Semen } 30 \mathrm{~g} \text {, Astragali Radix } 30 \mathrm{~g} \text {, Scapharcae seu } \\
\text { Tegillarcae Concha } 15 \mathrm{~g} \text {, Litchi Semen } 15 \mathrm{~g} \text {, Cremastrae } \\
\text { Tuber } 10 \mathrm{~g} \text {, Agastachis Herba } 9 \text { g, Scolopendra two pieces, } \\
\text { Draconis Sanguis } 6 \text { g, Eupolyphaga } 6 \text { g, Olibanum } 6 \text { g, } \\
\text { Myrrha } 6 \text { g, Glycyrrhizae Radix et Rhizoma } 6 \mathrm{~g}\end{array}$ & Eupolyphaga, Scolopendra & One dose per day, bid & n.r. \\
\hline 8 & $\begin{array}{l}\text { Lin } \\
(2011) \\
{[42]}\end{array}$ & $\begin{array}{l}\text { Whole scorpion bergamot } \\
\text { powder } \\
\text { (Quanxie Foshou san) }\end{array}$ & $\begin{array}{l}\text { Scorpio } 6 \text { g, Angelicae Gigantis Radix } 15 \text { g, Cnidii } \\
\text { Rhizoma } 10 \text { g, Leonuri Herba } 15 \text { g, Cyperi Rhizoma } 15 \text { g }\end{array}$ & Scorpio & One dose per day, n.r. & $\begin{array}{l}\text { From 3-4 days before } \\
\text { menstruation }\end{array}$ \\
\hline
\end{tabular}


Table 4. Cont.

\begin{tabular}{|c|c|c|c|c|c|c|}
\hline Study ID & $\begin{array}{c}\text { Author } \\
\text { (Year) }\end{array}$ & Herbal Medicine & Component & $\begin{array}{l}\text { Toxic Animal-Based } \\
\text { Medicinal Materials }\end{array}$ & Dosage, Frequency & Dosing Period \\
\hline 9 & $\begin{array}{c}\text { Meng } \\
(2012) \\
{[43]}\end{array}$ & A n.r. & $\begin{array}{c}\text { Hirudo 1-2 g } \\
\text { Angelicae Gigantis Radix 10-15 g, Cnidii Rhizoma 6-10 g, } \\
\text { Leonuri Herba } 15 \text { g, Paeoniae Radix Rubra } 10 \text { g, Paeoniae } \\
\text { Radix Alba } 10 \text { g, Curcumae Longae Rhizoma } 10 \text { g, } \\
\text { Sparganii Rhizoma } 10 \text { g, Curcumae Rhizoma } 10 \text { g, } \\
\text { Sargentodoxa cuneata } 30 \text { g, Sepiae Endoconcha } 12 \text { g }\end{array}$ & Hirudo & n.r., tid & $\begin{array}{l}\text { From 3-4 days before } \\
\text { menstruation to the end } \\
\text { of menstruation }\end{array}$ \\
\hline 10 & $\begin{array}{c}\text { Guo } \\
(2013) \\
{[44]}\end{array}$ & $\begin{array}{l}\text { Bushen Quyu Decoction } \\
\text { (Bushen Quyu tang) }\end{array}$ & $\begin{array}{l}\text { Lycii Fructus } 15 \text { g, Rehmanniae Radix Preparata } 15 \text { g, } \\
\text { Ligustri Lucidi Fructus } 15 \text { g, Cuscutae Semen } 15 \text { g, } \\
\text { Angelicae Gigantis Radix } 10 \text { g, Paeoniae Radix Rubra } \\
15 \text { g, Paeoniae Radix Alba } 15 \text { g, Achyranthis Radix } 12 \text { g, } \\
\text { Bupleuri Radix } 10 \text { g, Sappan Lignum } 10 \text { g, Sparganii } \\
\text { Rhizoma } 10 \text { g, Curcumae Rhizoma } 10 \text { g, Hirudo } 10 \text { g }\end{array}$ & Hirudo & One dose per day, bid & From MCD 1 \\
\hline 11 & $\begin{array}{c}\text { Liu } \\
(2017) \\
{[45]}\end{array}$ & $\begin{array}{l}\text { Tongjingxiao Granules } \\
\text { (Tongjingxiao keli) }\end{array}$ & $\begin{array}{c}\text { Salviae Miltiorrhizae Radix } 20 \mathrm{~g} \text {, Paeoniae Radix Rubra } \\
20 \mathrm{~g} \text {, Angelicae Gigantis Radix } 10 \mathrm{~g} \text {, Scolopendra } 6 \mathrm{~g} \text {, } \\
\text { Corydalis Tuber } 20 \mathrm{~g} \text {, Meliae Fructus } 10 \mathrm{~g} \text {, Glycyrrhizae } \\
\text { Radix et Rhizoma } 6 \mathrm{~g}\end{array}$ & Scolopendra & One dose per day, bid & $\begin{array}{l}\text { From } 7 \text { days before } \\
\text { menstruation to the end } \\
\text { of menstruation }\end{array}$ \\
\hline 12 & $\begin{array}{c}\mathrm{Yi} \\
(2018) \\
{[46]}\end{array}$ & $\begin{array}{l}\text { Xiaoyi Analgesic Soup } \\
\text { (Xiaoyi Zhitong tang) }\end{array}$ & $\begin{array}{c}\text { Cinnamomi Ramulus } 20 \text { g, Poria Sclerotium } 30 \text { g, Salviae } \\
\text { Miltiorrhizae Radix } 20 \text { g, Persicae Semen } 20 \text { g, Curcumae } \\
\text { Rhizoma } 10 \text { g, Scolopendra two pieces, Eupolyphaga } \\
10 \text { g, Hirudo } 10 \text { g, Pelodiscis Carapax } 20 \mathrm{~g}\end{array}$ & $\begin{array}{l}\text { Eupolyphaga, Hirudo, } \\
\text { Scolopendra }\end{array}$ & n.r., bid & $\begin{array}{l}\text { From the end of } \\
\text { menstruation }\end{array}$ \\
\hline
\end{tabular}

n.r., not reported; bid, twice a day; tid, three times a day; MCD, menstrual cycle day. 


\section{Discussion}

In this scoping review, we outlined preclinical and clinical studies of toxic animalbased medicinal materials for the treatment of endometriosis. By reviewing the experimental and clinical studies included in this study, we identified the potential of toxic animal-based medicinal materials as a treatment of endometriosis. However, the current evidence is insufficient for establishing a standard for endometriosis treatment with toxic animal-based medicinal materials. High-quality studies are needed to investigate the various action mechanisms of toxic animal-based medical materials against endometriosis.

Even though we did not impose any restrictions in the literature search, we found more studies published in China than in any other country. Traditional Korean medicine and traditional Chinese medicine were alike as both are recognized by the healthcare systems of those counties. However, in the healthcare systems of the two countries, traditional Chinese medicine has a bigger share than that of traditional Korean medicine. Furthermore, traditional Chinese medicine spans a broader range, as it includes not only traditional Chinese medicine but also the integration of Chinese and Western medicine [47]. Therefore, it is believed that there would be more cases of using toxic animal-based medicinal materials in clinical contexts in China, with more reports for that matter.

The toxic animal-based medical materials investigated in the preclinical and clinical studies included in this analysis were somewhat different. Hirudo and Scolopendra were used in both preclinical and clinical studies. In contrast, Eupolyphaga and Scorpio were used only in clinical studies, and bufalin, bee venom, and snake venom were used only in preclinical studies. This is because Bufonis Venenum, bee venom, and snake venom are animal-derived toxins that can cause serious side effects, including death [22,48-51], and thus have not been traditionally used to treat endometriosis and benign disease. Although these three animal venoms have recently been reported to exert proapoptotic [52-54] and anti-inflammatory effects $[55,56]$, there has been no clinical evidence of their benefit in endometriosis treatment. These three venoms are thought to be effective against endometriosis because they have been reported to induce apoptosis, inhibit angiogenesis, reduce estrogen levels, suppress MMP-9 expression, and exert anti-inflammatory effects in preclinical studies. However, considering the serious side effects that may occur, the in vitro and in vivo toxicity and action mechanisms of bufo venom, bee venom, and snake venom must be elucidated before their efficacy against endometriosis can be investigated in clinical trials.

The 12 clinical studies included in this study reported improvement of various endometriosis symptoms, such as dysmenorrhea, pelvic pain, dyspareunia, mittelschmerz, anal pain, as well as increased pregnancy rate after administration of a decoction containing Hirudo, Eupolyphaga, Scolopendra, and Scorpio. However, as there were differences in the symptoms, dosage, and dosing period of the toxic animal-based medicinal materials used, the evidence was considered insufficient for establishing a standard for the application of toxic animal-based medicinal materials in the treatment of endometriosis. In future studies, the optimal dosage of the medicines should be established with consideration for both efficacy and toxicity, and clinical studies must be conducted to standardize the dosing period and dosage.

The most widely used toxic animal-based medicinal material among the included clinical studies was Hirudo Figure 2. Hirudo is the dried entire body of Whitmania pigra Whitman, Hirudo nipponica Whitman, or W. acranulata Whitman, and is a representative toxic animal-based medicinal material that has been used since the beginning of civilization. It has the efficacy of "breaking blood and expelling stasis", and there is historical evidence of its use in the treatment of endometriosis $[22,57,58]$. However, it is difficult to confirm the effectiveness of Hirudo against endometriosis, as there were no studies examining only Hirudo. Nevertheless, a medicinal decoction containing Hirudo was shown to exert therapeutic effect against endometriosis in both preclinical and clinical studies. 


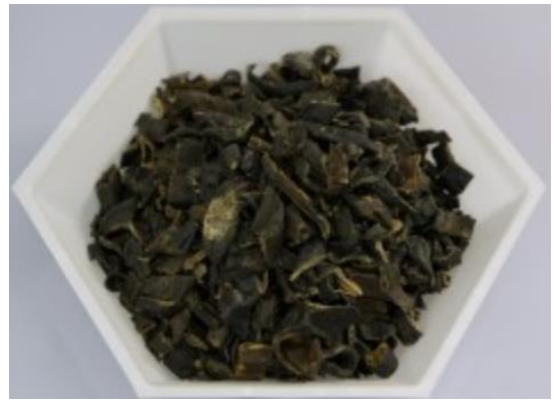

(A) Hirudo

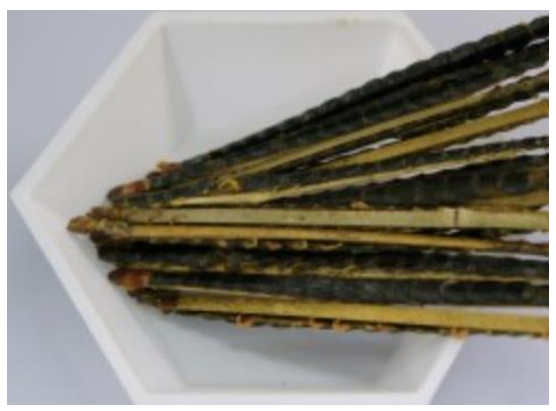

(C) Scolopendra

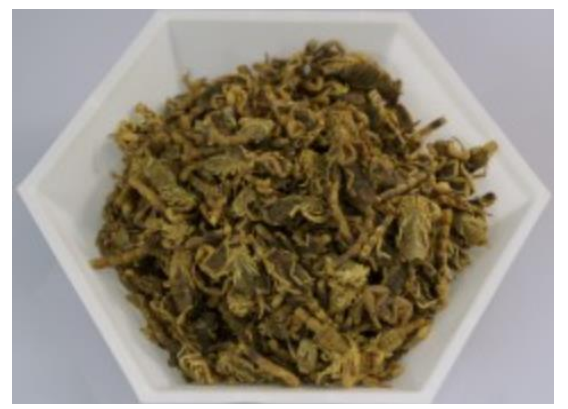

(B) Scorpio.

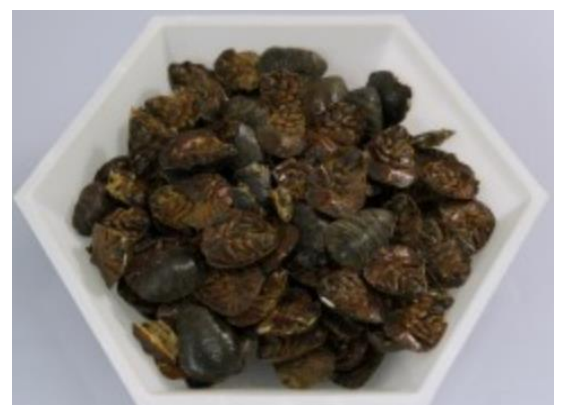

(D) Eupolyphaga

Figure 2. Toxic animal-based medicinal material in the includes clinical studies. (A) Hirudo: The dried entire body of Whitmania pigra Whitman, Hirudo nipponica Whitman, or W. acranulata Whitman. (B) Scorpio: The dried body of Buthus martensii Karsch scorpion. (C) Scolopendra: The dried body of Scolopendra subspinipes mutilans L. Koch. (D) Eupolyphaga: The dried body of female Eupolyphaga sinensis Walker or Steleophaga plancyi (Boleny).

In the studies included in this scoping review, medicinal decoctions containing Hirudo was shown to downregulate the expression of hypoxia-inducible factor 1-alpha (HIF-1 $\alpha)$ and reduce the level of vascular endothelial growth factor (VEGF), thereby suppressing the progression of endometriosis [32]. They also reduced the levels of basic fibroblast growth factor, platelet-derived growth factor, and VEGF, and were involved in the angiogenesis pathway by downregulating TLR4 and NF- $\mathrm{kB}$, which participate in the signaling process of angiogenesis, thus improving endometriosis [31]. It was also suggested that a medical decoction containing Hirudo exerted anti-inflammatory effect [33]. These findings are supported by other experimental studies. Hirudo has been revealed to have anticoagulant, anti-inflammatory, bacteriostatic, and analgesic effects [22,59]. Hirudo is also known to inhibit tumor angiogenesis by improving the tumor hypoxia microenvironment, reducing the mRNA and protein expression of HIF- $1 \alpha$, and contributing to the downregulation of VEGF mRNA expression [60]. In addition, Hirudo induces apoptosis and inhibits cell proliferation in Hep G2 human liver cancer cells and HL-60 leukemic cells, resulting in an antitumor effect $[61,62]$. Although the use of Hirudo is controversial, Hirudo exerts anti-inflammatory effect by inhibiting carboxypeptidases (kininase 1), inhibits platelet function, exerts anticoagulant activity, and increases blood flow [63].

Scolopendra was the second-most widely used toxic animal-based medicinal material in the included clinical studies. Scolopendra is the dried body of Scolopendra subspinipes mutilans L. Koch, which is poisonous. It has the efficacy of "extinguishing wind and suppressing convulsion, unblocking collaterals and relieving pain, counteracting toxins, and dissipating masses" and is used for "spasm and convulsion due to internal stirring of liver wind, etc." [57]. Medicinal decoctions containing Scolopendra were shown to be effective in treating endometriosis in both clinical and preclinical studies included in the present review. 
Experimental studies included in this study suggested that medical decoctions containing Scolopendra may exert therapeutic effect against endometriosis, which is a chronic inflammatory condition, through anti-inflammatory action [33]. This is supported by other studies, in which Scolopendra was reported to have anticoagulation, antiseptic, and antiinflammatory effects [64-66]. Scolopendra is also known to induce the downregulation of matrix metallopeptidase-2 (MMP-2) and -9 in tumor cells, suppress the migration and invasion of tumor cells, and inhibit cell proliferation [67]. In addition, Scolopendra inhibits the proliferation of EGFR-overexpressing cells by inducing apoptosis and modulating the EGFR pathway $[68,69]$. It also exerts anti-inflammatory activity partially through inhibition of the NF- $\mathrm{kB}$ signaling pathway [70].

Eupolyphaga, the third-most commonly used toxic animal-based medicinal material in this review, is the dried body of female Eupolyphaga sinensis Walker or Steleophaga plancyi (Boleny), which is slightly poisonous. It has the efficacy of "breaking blood and expelling stasis" and has been used clinically for "aggregation-accumulation due to static blood obstruction" [57]. Clinical studies in this review revealed that medicinal decoctions containing Eupolyphaga were effective in alleviating endometriosis symptoms. However, there have been no experimental studies that elucidate the anti-endometriosis mechanism of Eupolyphaga. Nevertheless, in other studies, Eupolyphaga was shown to inhibit cell adhesion to fibronectin and collagen IV as well as cell migration and invasion in A549 human lung cancer cell [71]. It also inhibits cell proliferation and reduces MMP-2 and -9 expression in hepatocellular carcinoma [72], and reduces cell invasive ability by downregulating MMP-2 and -9 protein expression in breast cancer [73]. Furthermore, it induces the detachment and apoptosis of A549 human lung cancer cells [71], inhibits tumor cell growth in hepatocarcinoma, promotes TNF- $\alpha$ and IFN- $\gamma$ expression, increases the Bax/Bcl-2 ratio, and activates caspases-3 to induce apoptosis [74]. Moreover, Eupolyphaga is known to activate the immune function by modulating oxidative systems, enhancing the phagocytic function of macrophages, and elevating serum IL-2 level [75].

Lastly, the dried body of Buthus martensii Karsch scorpion is known to be poisonous and exert liver-pacifying and wind-extinguishing effects. Scorpio has pharmacological properties, such as pain-reducing, anti-inflammatory, and anticoagulant properties [76,77], but no experimental studies have determined the therapeutic mechanism of Scorpio against endometriosis. Scorpio exerts an antitumor effect by inhibiting cell proliferation, inducing apoptosis, and decreasing migration and invasive functions in Hepa 1-6 cells and HepG2 cells $[78,79]$. They also inhibit MMP activity in breast and colorectal cancers, thereby reducing the motility and invasion of tumor cells, and induce apoptosis by decreasing the expression of antiapoptotic proteins and increasing that of proapoptotic proteins [80]. In addition, chlorotoxin derived from scorpion toxin inhibits the expression of ER $\alpha$ in breast cancer cells by inhibiting the ER $\alpha$ signaling pathway through direct binding to $E R \alpha$, thus changing the protein secondary structure of its LBD domain [81].

As outlined above, toxic animal-based medicinal materials are composed of several molecules that exhibit a wide range of pharmacological activities. Considering that endometriosis has several possible mechanisms of pathogenesis, multi-target medicines have the potential to treat endometriosis through multiple pathways. Further research is needed to elucidate the specific therapeutic mechanisms of individual toxic animal-based medicinal materials against endometriosis. Although the mechanisms underlying the progression of endometriosis are still unclear, endocrine, immunologic, pro-inflammatory, and pro-angiogenic processes are known to be involved in the development of endometriosis. Considering the pharmacological effects of toxic animal-based medicinal materials on endometriosis and other diseases, the possible action mechanisms of toxic animal-based medicinal materials against endometriosis can be summarized as follows: induction of apoptosis, antiangiogenesis effect, reduction of estrogen level, anti-inflammatory action, and suppression of cell adhesion and invasion (Figure 3). 


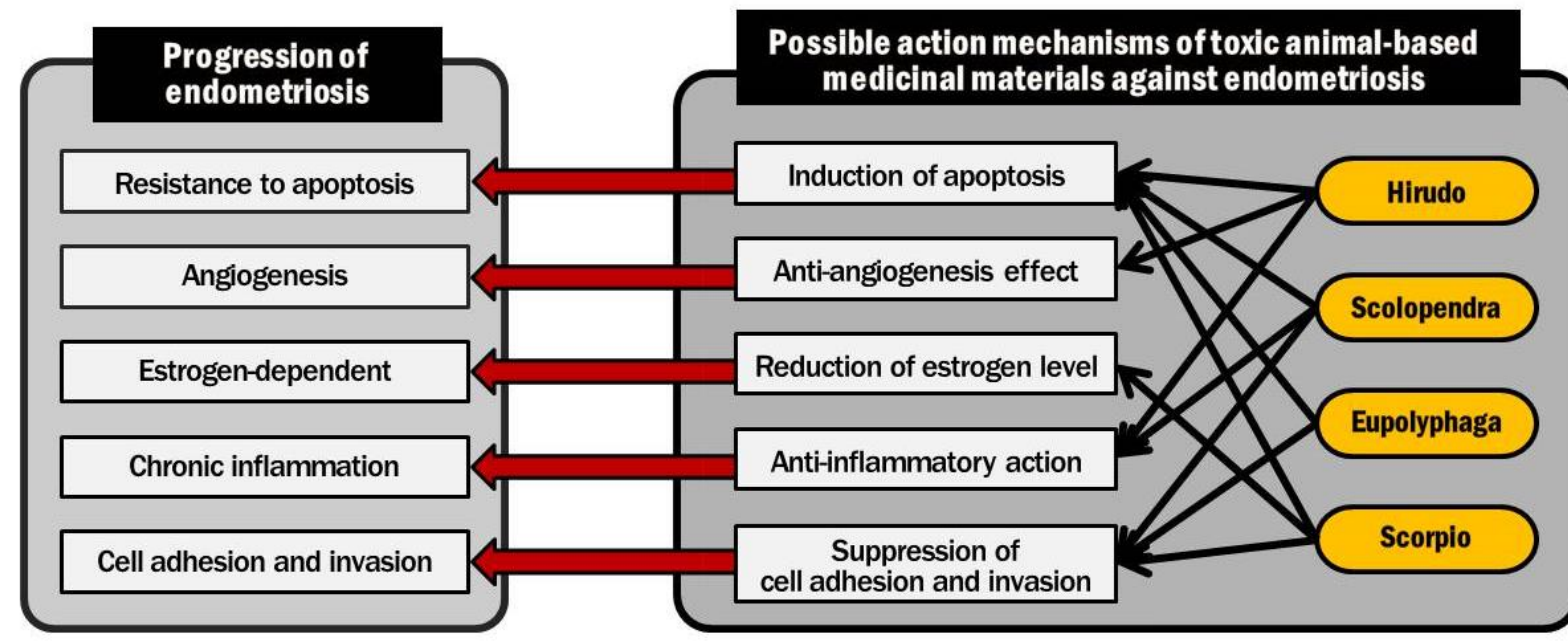

\section{Characteristics of Endometriosis}

\section{Possible Action Mechanisms of Toxic Animal-based Medicinal Materials against Endometriosis}

Figure 3. Possible action mechanisms of toxic animal-based medicinal materials against endometriosis.

Toxic animal-based medicinal materials may cause unwanted side effects due to their toxic effects. Minimizing adverse events is an essential part of drug development. Even with the medicines that have been used based on historical experience, the types and frequency of adverse events must be identified. However, among the studies included in this review, $75 \%$ of the studies had no reports on side-effects, and three studies $[37,42,45]$ reported that no side effects were observed after administration of herbal medicines containing Hirudo and Scolopendra [37], Scolopendra [45], and Scorpio [42]. However, it was not possible to find any information on what kind of events they were monitoring.

The number of participants who participated in the studies that reported no side effects was small. Since the known occurrence of adverse events was 5\% to 10\% [82,83], the findings of this study are not sufficient to support a conclusion that the administration of toxic animal-based medicinal materials upon the patients with endometriosis would result in no side effects. Even if the toxic animal-based medicinal materials have historical evidence, it is necessary to identify the existence, occurrence rate, and severity of adverse events through real data collection. A clinical study must be designed to report any adverse events, no matter how insignificant they may look.

In addition, in order to identify the possible adverse events that can be caused by toxic animal-based medicinal materials, we searched other studies that reported adverse events after administering toxic animal-based medicinal materials. Among the studies included in this review, three studies $[37,42,45]$ reported that no side effects were observed after administration of herbal medicines containing Hirudo and Scolopendra [37], Scolopendra [45], and Scorpio [42], and that no adverse effects were reported following the administration of herbal medicines containing Eupolyphaga. However, these findings are inconsistent with those of other studies. Hirudo is prohibited for pregnant women, patients with profuse menstruation or hemorrhagic tendency, patients with no blood stasis, and weak patients because Hirudo is highly potent in breaking blood and expelling stasis, unblocking the meridian, and eliminating mass [26,57]. Zeng et al. reported gastrointestinal bleeding $(1.9 \%)$ and cerebral hernia $(0.9 \%)$ after the administration of herbal medicines containing Hirudo and Tabanus bivittatus Matsumura in patients with acute intracerebral hemorrhage [84]. Historically, owing to its toxicity, Scolopendra has not been used at a high dosage, as its excessive use can damage healthy qi and cause early abortion [26]; therefore, it is prohibited for use in pregnant women and patients with blood deficiency generating wind [57]. In one patient with chronic hepatitis B infection, hepatotoxicity was reported after the administration of herbal medicine containing Scolopendra [85]. How- 
ever, this herbal medicine contained not only Scolopendra but also the hepatotoxic Meliae Fructus and Rhei Radix et Rhizoma [85]; these toxic effects cannot be attributed solely to Scolopendra, but caution is needed when subjecting patients with chronic hepatitis B to Scolopendra treatment. In traditional Chinese medicine, Eupolyphaga is contraindicated for pregnant women because it may induce early abortion owing to its blood-breaking and stasis- expelling efficacies, and it should be used with caution in patients with no blood stasis or patients with both blood deficiency and blood stasis [26]. Several studies have reported the side effects of Eupolyphaga. However, in a study where 21 HIV / AIDS patients were treated with a drug containing $20 \mathrm{~g}$ of Eupolyphaga twice a day for 4 months, no side effects or toxic reactions were reported [86]. Scorpion should be prohibited in patients with blood deficiency generating wind, and should be used at a dose range of $3-5 \mathrm{~g}$ owing to its toxicity [26,57]. There was one case of hepatotoxicity after administration of herbal medicine containing Scorpio in a patient with chronic hepatitis B infection [85]. Taken together, the patient's conditions should be considered when administering toxic animal-based medicinal materials, and attention should be paid to the menstrual cycle during treatment with these materials, as they can cause excessive bleeding in patients with menorrhagia. In addition, when administering these medicinal materials to women of childbearing age, the possibility of pregnancy should be assessed. Moreover, overuse of these medicinal materials should be avoided because it has been reported to be associated with hepatitis.

This is the first study to review both preclinical and clinical studies investigating the efficacy of toxic animal-based medicinal materials in the treatment of endometriosis. Our analysis results suggested the possible therapeutic mechanisms of toxic animal-based medical materials against endometriosis and provided data as a reference for future translational studies. In addition, we included studies from major databases, such as PubMed and Embase, and searched without language and date restrictions. However, there were some limitations in this review. First, as we used only the biopharmaceutical names as search terms, there is a possibility that potentially relevant articles not containing our search key words and phrases in the titles or abstracts were not included in our analysis. Second, the number of included studies was small. Third, it was difficult to determine whether the reported therapeutic effects were exerted by the toxic animal-based medicinal materials, as these materials were used concomitantly with other medicinal materials. Fourth, there were some factors that required attention in interpreting the study findings. Five of the clinical studies we reviewed were case series, and therefore the level of evidence was somewhat low. Seven RCTs did not provide their protocols and were without any description of their randomization or blinding methods. Furthermore, some studies used mifepristone or different types of herbal medicines rather than the conventional treatment of endometriosis as control, so caution is needed as one interprets the results of these studies. Some studies did not report side effects or what kind of important harms or unintended effects they observed. Therefore, even if some studies did not report side effects, it does not necessarily support the conclusion that toxic animal-based medicinal materials are safe to use. Lastly, endometriosis is diagnosed by ascertaining symptoms, undertaking examination, and performing laparoscopic surgery [87]. Of the studies that were included in this review, only three $[32,34,35]$ used laparoscopes to diagnose endometriosis. Other studies all relied on presumed diagnosis based on symptoms, examination, ultrasonography, and lab test results, without any classification of endometriosis, which were the limitations of these studies.

Through this scoping review, we showed that toxic animal-based medicinal materials might be effective as a clinical treatment of endometriosis. However, because the number of clinical studies included was small and the intervention and treatment goals were different, the findings of this review are insufficient for establishing a standard for the application of toxic animal-based medicinal materials as a treatment of endometriosis. In addition, although toxic animal-based medicinal materials have been shown to be effective against endometriosis in experimental studies, research on their specific therapeutic mechanisms 
is still lacking. Therefore, evidence supporting the use of toxic animal-based medicinal materials as a treatment of endometriosis is still limited, and further research is necessary.

The preclinical studies and clinical studies included in this review showed that the most promising toxic animal-based medicinal material for endometriosis is likely to be Hirudo. As shown in Figure 3, Hirudo, Scolopendra, and Scorpio meet three categories of action mechanisms related to the progression of endometriosis. Eupolyphaga meets two categories. In addition, in the clinical studies shown in Table 3, Hirudo was included in eight studies. Scolopendra, Eupolyphaga, and Scoprpio were included in five, four, and one study, respectively. Thus, we assume that Hirudo might be the most prominent candidate for treating endometriosis, among these toxic animal-based medicinal materials. The preclinical studies demonstrated that Hirudo has activities inducing apoptosis and inhibiting angiogenesis and inflammation. Although these molecular mechanisms are closely related to the progression of endometriosis, the anti-endometriotic efficacy of Hirudo was not fully demonstrated by a clinical study. In addition, there were no studies that used Hirudo only, so further study is needed to examine the treatment effect of Hirudo upon endometriosis. Scorpio, Scolopendra, and Eupholyphaga have been in clinical use, but their preclinical study is not sufficient. Bee venom, Bufonis Venenum, and snake venom have been used in preclinical contexts, without any report of toxicity. As bee venom, Bufonis Venenum, and snake venom may cause a serious adverse event, it is necessary to identify the adverse events and appropriate doses via preclinical studies.

In the future, the following steps would have to be taken in order to develop a new drug for endometriosis based on toxic animal-based medicinal materials. First, classical literature and medical records should be searched to obtain additional evidence supporting the use of toxic animal-based medicinal materials in clinical setting. Next, extensive preclinical studies are needed to elucidate the mechanisms of endometriosis treatment that are frequently used in clinical practice. Research is needed not only on the therapeutic mechanism of a single toxic animal-based medicinal material against endometriosis but also on the interaction between medicinal materials and decoctions that are traditionally used in combinations, including toxic animal-based medicinal materials. As they have toxic properties, quality control of single toxic animal-based medicinal materials is necessary, and it is important to assess their safety and toxicity in future studies. Additional clinical trials should be conducted to establish the standardized as well as optimal dosage and dosing period of these medicinal materials.

\section{Conclusions}

In this scoping review, preclinical and clinical studies investigating the efficacy of toxic animal-based medicinal materials against endometriosis did not provide sufficient evidence to allow standardization of these materials as a treatment of endometriosis. However, the included studies have identified toxic animal-based medical materials as potential treatments for endometriosis. In clinical studies, the administration of herbal medicines containing toxic animal-based medicinal materials alleviated symptoms such as dysmenorrhea, infertility, endometrioma, and menstrual irregularities in patients with endometriosis, with no side effects. In experimental studies, the main action mechanisms of toxic animalbased medicinal materials against endometriosis were identified as induction of apoptosis, antiangiogenesis effect, reduction of estrogen levels, and possibly, anti-inflammatory action. Taken together, toxic animal-based medicinal materials are considered promising to be developed as an effective treatment of endometriosis; however, additional studies are needed.

Author Contributions: Conceptualization, S.-I.H. and J.-K.P.; methodology, S.-H.S. and J.-K.P.; data classification and interpretation of data, S.-I.H., S.-H.S., Y.-J.Y., and K.-T.H.; writing-original draft, S.-I.H.; writing-review and editing, S.-H.S, K.-T.H, and J.-K.P.; supervision, J.-K.P.; project administration, J.-K.P.; funding acquisition, J.-K.P. All authors have read and agreed to the published version of the manuscript. 
Funding: This research was supported by the National Research Foundation of Korea (NRF) grant funded by the Korean government (MSIT) (No. NRF-2019R1G1A1100022).

Institutional Review Board Statement: Not applicable.

Informed Consent Statement: Not applicable.

Data Availability Statement: The data will be made available upon reasonable request.

Acknowledgments: Picture of Hirudo, Scorpio, Scolopendra, and Eupolyphaga used in this review were prepared by the Koran Institute of Oriental Medicine in 2017, disclosed for open access under Type 1 KOGL (Korea Open Government Licenses). The said copyright material can be download at Korea Institute of Oriental Medicine, https:/ / herba.kr/boncho/?m=list\&t=pics $\backslash \mathrm{T} 1 \backslash$ textquoteright free of charge.

Conflicts of Interest: The authors declare that there are no conflict of interest regarding the publication of this paper.

\section{References}

1. Bulun, S.E.; Yilmaz, B.D.; Sison, C.; Miyazaki, K.; Bernardi, L.; Liu, S.; Kohlmeier, A.; Yin, P.; Milad, M.; Wei, J. Endometriosis. Endocr. Rev. 2019, 40, 1048-1079. [CrossRef]

2. Zondervan, K.T.; Becker, C.M.; Koga, K.; Missmer, S.A.; Taylor, R.N.; Viganò, P. Endometriosis. Nat. Rev. Dis. 2018, 4, 9. [CrossRef]

3. Shafrir, A.L.; Farland, L.V.; Shah, D.K.; Harris, H.R.; Kvaskoff, M.; Zondervan, K.; Missmer, S.A. Risk for and consequences of endometriosis: A critical epidemiologic review. Best Pract. Res. Clin. Obstet. Gynaecol. 2018, 51, 1-15. [CrossRef]

4. Zondervan, K.T.; Becker, C.M.; Missmer, S.A. Endometriosis. N. Engl. J. Med. 2020, 382, 1244-1256. [CrossRef]

5. Falcone, T.; Flyckt, R. Clinical Management of Endometriosis. Obstet. Gynecol. 2018, 131, 557-571. [CrossRef]

6. Peiris, A.N.; Chaljub, E.; Medlock, D. Endometriosis. JAMA 2018, 320, 2608. [CrossRef] [PubMed]

7. Czyzyk, A.; Podfigurna, A.; Szeliga, A.; Meczekalski, B. Update on endometriosis pathogenesis. Minerva Ginecol. 2017, 69, 447-461. [CrossRef] [PubMed]

8. Flores, I.; Rivera, E.; Ruiz, L.A.; Santiago, O.I.; Vernon, M.W.; Appleyard, C.B. Molecular profiling of experimental endometriosis identified gene expression patterns in common with human disease. Fertil. Steril. 2007, 87, 1180-1199. [CrossRef] [PubMed]

9. Van Gorp, T.; Amant, F.; Neven, P.; Vergote, I.; Moerman, P. Endometriosis and the development of malignant tumours of the pelvis. A review of literature. Best Pract. Res. Clin. Obstet. Gynaecol. 2004, 18, 349-371. [CrossRef] [PubMed]

10. Krupa, A.; Padała, O.; Putowski, M.; Konopelko, M.; Piasek, E. Available treatment methods for endometriosis. J. Educ. Health Sport. 2019, 9, 178-184. [CrossRef]

11. Practice Committee of the American Society for Reproductive Medicine. Treatment of pelvic pain associated with endometriosis: A committee opinion. Fertil. Steril. 2014, 101, 927-935. [CrossRef] [PubMed]

12. Bina, F.; Soleymani, S.; Toliat, T.; Hajimahmoodi, M.; Tabarrai, M.; Abdollahi, M.; Rahimi, R. Plant-derived medicines for treatment of endometriosis: A comprehensive review of molecular mechanisms. Pharmacol. Res. 2019, 139, 76-90. [CrossRef] [PubMed]

13. Meresman, G.F.; Götte, M.; Laschke, M.W. Plants as source of new therapies for endometriosis: A review of preclinical and clinical studies. Hum Reprod Update. 2020, dmaa039. [CrossRef] [PubMed]

14. Zheng, W.; Wu, J.; Gu, J.; Weng, H.; Wang, J.; Wang, T.; Liang, X.; Cao, L. Modular Characteristics and Mechanism of Action of Herbs for Endometriosis Treatment in Chinese Medicine: A Data Mining and Network Pharmacology-Based Identification. Front. Pharmacol. 2020, 11, 147. [CrossRef] [PubMed]

15. Han, Y.F.; Hou, L.H.; Zhou, Y.J.; Wu, X.K. A survey of TCM treatment for endometriosis. J. Tradit. Chin. Med. 2009, 29, 64-70. [CrossRef]

16. Bae, K.Y.; Rhyu, M.R.; Roh, J.J.; Kim, D.I. Vasodilatory Activities and Safety of the Water Extracts of Three Medicinal Remedy in Species of Insects. J. Korean Obstet. Gynecol. 2007, 20, 114-124.

17. Sha, B.; Zhu, W.H. On the Application of Insect Medicine in Rheumatism from the Inheritance of Menghe Medical School. Rheumatism Arthritis 2020, 8, 64-65.

18. Wang, W.P.; Zou, J.; Wu, W. Case Records Illustration of Professor WU Wei Who has Used Insect Medicine to Treat Kidney Disease. J. Sichuan Tradit. Chin. Med. 2020, 7, 85-87.

19. Chen, Y.; Ji, L.; Wang, M.; Li, M.; Cui, S. Research Progress in the Application of Insect Medicinals in Gynecological Diseases. Shandong J. Tradit. Chin. Med. 2020, 9, 1022-1025.

20. Wang, J.H.; Wang, T.Z.; Jiang, D.Y. JIANG De-you's experience in treating cardiovascular and cerebrovascular diseases with insect drug. China J. Tradit. Chin. Med. Pharm. 2020, 8, 3962-3965.

21. Klupczynska, A.; Pawlak, M.; Kokot, Z.J.; Matysiak, J. Application of Metabolomic Tools for Studying Low Molecular-Weight Fraction of Animal Venoms and Poisons. Toxins 2018, 10, 306. [CrossRef] [PubMed]

22. Bordon, K.C.F.; Cologna, C.T.; Fornari-Baldo, E.C.; Pinheiro-Júnior, E.L.; Cerni, F.A.; Amorim, F.G.; Anjolette, F.A.P.; Cordeiro, F.A.; Wiezel, G.A.; Cardoso, I.A.; et al. From Animal Poisons and Venoms to Medicines: Achievements, Challenges and Perspectives in Drug Discovery. Front. Pharmacol. 2020, 11, 1132. [CrossRef] 
23. Chatterjee, B. Animal Venoms have Potential to Treat Cancer. Curr. Top. Med. Chem. 2018, 18, 2555-2566. [CrossRef]

24. Ravi, K.U. Use of Animal Venom Peptides/Toxins in Cancer Therapeutics. Nat. Biomed. Eng. 2018, 16, 1-26. [CrossRef]

25. Choi, G. Animal-based medicinal materials in the pharmacopeias of Northeast Asian countries. Korean Herb. Med. Inf. 2018, 6, 203-230. [CrossRef]

26. Herbology editorial committee of Korean medicine schools. Herbology; Yeonglimsa: Seoul, Korea, 2012; pp. 469-471, 546-548.

27. Lim, E.-M.; Kwon, K.-R.; Lee, Y.-H. Effects of Bee Venom Acupuncture on Surgically Induced Endometriosis Rats. J. Pharmacopunct. 2006, 9, 21-32.

28. Nasu, K.; Nishida, M.; Ueda, T.; Takai, N.; Bing, S.; Narahara, H.; Miyakawa, I. Bufalin induces apoptosis and the G0/G1 cell cycle arrest of endometriotic stromal cells: A promising agent for the treatment of endometriosis. Mol. Hum. Reprod. 2005, 11, 817-823. [CrossRef]

29. Cho, Y.J.; Lee, J.E.; Park, M.J.; O’Malley, B.W.; Han, S.J. Bufalin suppresses endometriosis progression by inducing pyroptosis and apoptosis. J. Endocrinol. 2018, 237, 255-269. [CrossRef]

30. Liu, Y.L. Investigate the role of "Pingchongjiangnifang" of the in vitro culture of human endometrial stromal cells. Master's Thesis, Jiangxi University of Traditional Chinese Medicine, Nanchang, China, 1 May 2014.

31. Xu, Z. Data Mining and Experimental Research on the Treatment of Endometriosis Dysmenorrhea by Professor Si Tuyi, a Famous Chinese Medicine Practitioner. Ph.D. Thesis, Guangzhou University of Chinese Medicine, Guangzhou, China, 1 April 2018.

32. Liu, H.; Sun, X.; Zhao, Y.; Xia, M.; Wang, C. Anti-angiogenesis effect and mechanism study of Huangzhi Neiyi capsule in a rat endometriosis model. J. Int. Med. Res. 2020, 48, 300060519899767. [CrossRef] [PubMed]

33. Wang, T.F. The Effect of Wudanwan Modified Formula on Rat Endometriosis Model. Master's Thesis, Beijing University of Chinese Medicine, Beijing, China, 1 May 2005.

34. Zhang, Q. Effects of Disintegrin of Gloydius Brevicaudus Venom on Endometriosis of Rat and Its Mechanism. Master's Thesis, Fujian Medical University, Fuzhou, China, 1 June 2015.

35. Wu, X. Treatment of 60 cases of endometriosis by dissolving blood and removing blood stasis. Shaanxi Tradit. Chin. Med. 1993, 14,530 .

36. Zhang, L. Professor Wang Ziyu's Experience in Treating Endometriosis Dysmenorrhea. Master's Thesis, Beijing University of Chinese Medicine, Beijing, China, 1 May 2007.

37. Xu, Y.; Su, J.; Cao, H. Shuizhi Tongluo Capsule in the treatment of 52 cases of endometriosis. Hebei Tradit. Chin. Med. 2007, 29, 797.

38. Zhang, X.; Wang, W. Clinical analysis of 78 cases of endometriosis treated with Quyu Jiedu Xiaozheng Decoction. Mod. Chin. Med. 2007, 27, 18-19.

39. Feng, D. Xiaoyi Zhuyun Decoction in the treatment of 35 cases of endometriotic infertility. Res. Chin. Med. 2014, $27,36-37$.

40. Han, D.; Yu, F.; Wang, H. Clinical observation on 78 cases of endometriosis treated with traditional Chinese medicine leech. Chin. J. Coal Ind. Med. 2009, 12, 303-304.

41. Zhang, X.; Dong, L.; Wang, Q. Treatment of 45 cases of endometriosis with Quyu Jiedu Xiaozheng Decoction. Shaanxi J. Tradit. Chin. Med. 2009, 30, 260-261.

42. Lin, H.; Xiao, W.; He, X.; Peng, J. Observation on the clinical efficacy of Quanxie Foshou Powder in the treatment of endometriosis. Lishizhen Med. Mater. Med. 2011, 22, 2819-2820.

43. Meng, W.; Xu, Y. Clinical observation of 156 cases of endometriosis treated with traditional Chinese medicine leech. J. Changchun Univ. Tradit. Chin. Med. 2012, 28, 128-129.

44. Guo, M.; Qi, S.; Niu, G.; Liu, Z. Bushen Quyu Decoction combined with desogestrel and ethinyl estradiol tablets in the treatment of 48 cases of infertility with endometriosis after laparoscopic surgery. J. Tradit. Chin. Med. 2013, 26, 26-28.

45. Liu, H. Self-Made Dysmenorrhea Particles in the Treatment of Dysmenorrhea Caused by Endometriosis (Qi and Blood Stasis Type) the Safety and Clinical Research. Master's Thesis, Changchun University of Traditional Chinese Medicine, Changchun, China, 1 March 2017.

46. Yi, S.; Liu, L. The clinical study of Xiaoyizhitong Decoction combined with gestrinone in the treatment of endometriosis. Chin. Med. Guide 2018, 16, 215-216.

47. Ko, C.R.; Ku, N.P.; Seol, S.S. A Comparative Study on the Traditional Medicine Policies between Korea and China: Focused on the Second Korean Medicine Development Plan and the 12.5 Traditional Chinese Medicine Development Plan. J. Korea Technol. Innov. Soc. 2014, 17, 421-447.

48. Wei, W.L.; Hou, J.J.; Wang, X.; Yu, Y.; Li, H.J.; Li, Z.W.; Feng, Z.J.; Qu, H.; Wu, W.Y.; Guo, D.A. Venenum bufonis: An overview of its traditional use, natural product chemistry, pharmacology, pharmacokinetics and toxicology. J. Ethnopharmacol. 2019, 237, 215-235. [CrossRef]

49. Jang, S.; Kim, K.H. Clinical Effectiveness and Adverse Events of Bee Venom Therapy: A Systematic Review of Randomized Controlled Trials. Toxins 2020, 12, 558. [CrossRef] [PubMed]

50. Wei, J.M.; Zhu, M.W.; Zhang, Z.T.; Jia, Z.G.; He, X.D.; Wan, Y.L.; Wang, S.; Xiu, D.R.; Tang, Y.; Li, J.; et al. A multicenter, phase III trial of hemocoagulase Agkistrodon: Hemostasis, coagulation, and safety in patients undergoing abdominal surgery. Chin. Med. J. 2010, 123, 589-593. [PubMed]

51. Xu, Y.Y.; Ma, X.H.; Zhang, S.J. Hemocoagulase agkistrodon-induced anaphylactic shock: A case report and literature review. Int. J. Clin. Pharmacol. Ther. 2016, 54, 129-134. [CrossRef] [PubMed] 
52. Miao, Q.; Bi, L.L.; Li, X.; Miao, S.; Zhang, J.; Zhang, S.; Yang, Q.; Xie, Y.H.; Zhang, J.; Wang, S.W. Anticancer effects of bufalin on human hepatocellular carcinoma HepG2 cells: Roles of apoptosis and autophagy. Int. J. Mol. Sci. 2013, 14, 1370-1382. [CrossRef]

53. Qi, F.; Li, A.; Inagaki, Y.; Kokudo, N.; Tamura, S.; Nakata, M.; Tang, W. Antitumor activity of extracts and compounds from the skin of the toad Bufo bufo gargarizans Cantor. Int. Immunopharmacol. 2011, 11, 342-349. [CrossRef]

54. Liu, L.; Chen, B.A.; Qin, S.K. Anti-angiogenesis effect of arsenic trioxide plus cinobufacin on human hepatocarcinoma transplantation model nude mice. Chin. J. Integr. Tradit. West. Med. 2011, 31, 67-72.

55. Lee, J.A.; Son, M.J.; Choi, J.; Jun, J.H.; Kim, J.I.; Lee, M.S. Bee venom acupuncture for rheumatoid arthritis: A systematic review of randomised clinical trials. BMJ Open 2014, 4, e006140. [CrossRef] [PubMed]

56. Gazerani, P.; Cairns, B.E. Venom-based biotoxins as potential analgesics. Expert Rev. Neurother. 2014, 14, 1261-1274. [CrossRef]

57. Xi, S.; Gong, Y. Essentials of Chinese Materia Medica and Medical Formulas: New Century Traditional Chinese Medicine; Elsevier (Academic Press): London, UK, 2017; pp. 246-319.

58. Lu, J.; Chen, X.; Xu, X.; Liu, J.; Zhang, Z.; Wang, M.; Li, X.; Chen, H.; Zhao, D.; Wang, J.; et al. Active polypeptides from Hirudo inhibit endothelial cell inflammation and macrophage foam cell formation by regulating the LOX-1/LXR- $\alpha /$ ABCA1 pathway. Biomed. Pharmacother. 2019, 115, 108840. [CrossRef] [PubMed]

59. Dong, H.; Ren, J.X.; Wang, J.J.; Ding, L.S.; Zhao, J.J.; Liu, S.Y.; Gao, H.M. Chinese Medicinal Leech: Ethnopharmacology, Phytochemistry, and Pharmacological Activities. Evid.-Based Complement. Altern. Med. 2016, 2016, 7895935. [CrossRef]

60. Wu, Q.; Guo, J.; Lu, H.; Kong, Q. Impacts of leech hirudo and blister beetle mylabris on the angiogenesis of chick chorioallantonic membrance. World J. Integr. Tradit. West. Med. 2010, 5, 855-857.

61. Guo, Y.; Tian, X.; Xiao, Z. Study on inhibition effects of freeze-thawing leech extract on HepG2 cells. Chin. J. Inf. Tradit. Chin. Med. 2009, 16, 30-31.

62. Xiao, Y.S.; Liao, F.S.; Zhao, Z.D.; Zuo, A.R.; Zhu, D.C. Study on inhibitory functions of leech extract on HL60 cells in vitro experiment. J. Jiangxi Univ. Tradit. Chin. Med. 2013, 25, 63-66.

63. Sig, A.K.; Guney, M.; Uskudar Guclu, A.; Ozmen, E. Medicinal leech therapy-an overall perspective. Integr. Med. Res. 2017, 6, 337-343. [CrossRef]

64. Lee, W.; Lee, J.; Kulkarni, R.; Kim, M.A.; Hwang, J.S.; Na, M.; Bae, J.S. Antithrombotic and antiplatelet activities of small-molecule alkaloids from Scolopendra subspinipes mutilans. Sci. Rep. 2016, 6, 21956. [CrossRef] [PubMed]

65. Park, Y.J.; Lee, H.Y.; Jung, Y.S.; Park, J.S.; Hwang, J.S.; Bae, Y.S. Antimicrobial peptide scolopendrasin VII, derived from the centipede Scolopendra subspinipes mutilans, stimulates macrophage chemotaxis via formyl peptide receptor 1. BMB Rep. 2015, 48, 479-484. [CrossRef]

66. Park, J.H.; Lee, S.R. Anti-inflammatory activities of Scolopendra subspinipes mutilans in RAW 264.7 cells. J. Nutr. Health 2018, 51, 323. [CrossRef]

67. Zheng, L.; He, H.; Shen, X.; Sun, Y. Centipede Scolopendra suppresses cell growth in human epidermoid carcinoma cell A431. Bangladesh J. Pharmacol. 2017, 12, 299. [CrossRef]

68. Ma, W.; Liu, R.; Qi, J.; Zhang, Y. Extracts of centipede Scolopendra subspinipes mutilans induce cell cycle arrest and apoptosis in A375 human melanoma cells. Oncol. Lett. 2014, 8, 414-420. [CrossRef]

69. Ma, W.; Zhang, D.; Zheng, L.; Zhan, Y.; Zhang, Y. Potential roles of Centipede Scolopendra extracts as a strategy against EGFR-dependent cancers. Am. J. Transl. Res. 2015, 7, 39-52.

70. Hwang, L.; Ko, I.G.; Jin, J.J.; Kim, S.H.; Kim, C.J.; Jeon, J.W.; Han, J.H. Scolopendra subspinipes mutilans Extract Suppresses Inflammatory and Neuropathic Pain In Vitro and In Vivo. Evid.-Based Complement. Altern. Med. 2018, 2018, 5057372. [CrossRef] [PubMed]

71. Wang, F.X.; Wu, N.; Wei, J.T.; Liu, J.; Zhao, J.; Ji, A.G.; Lin, X.K. A novel protein from Eupolyphaga sinensis inhibits adhesion, migration, and invasion of human lung cancer A549 cells. Biochem. Cell Biol. 2013, 91, 244-251. [CrossRef]

72. Zhang, Y.; Zhan, Y.; Zhang, D.; Dai, B.; Ma, W.; Qi, J.; Liu, R.; He, L. Eupolyphaga sinensis Walker displays inhibition on hepatocellular carcinoma through regulating cell growth and metastasis signaling. Sci. Rep. 2014, 4, 5518. [CrossRef]

73. Zhan, Y.; Zhang, H.; Liu, R.; Wang, W.; Qi, J.; Zhang, Y. Eupolyphaga sinensis Walker ethanol extract suppresses cell growth and invasion in human breast cancer cells. Integr. Cancer Ther. 2016, 15, 102-112. [CrossRef]

74. Ge, G.F.; Yu, C.H.; Yu, B.; Shen, Z.H.; Zhang, D.L.; Wu, Q.F. Antitumor effects and chemical compositions of Eupolyphaga sinensis Walker ethanol extract. J. Ethnopharmacol. 2012, 141, 178-182. [CrossRef] [PubMed]

75. Liu, H.; Yan, Y.; Zhang, F.; Wu, Q. The immuno-enhancement effects of tubiechong (Eupolyphaga sinensis) lyophilized powder in cyclophosphamide-induced immunosuppressed mice. Immunol. Investig. 2019, 48, 844-859. [CrossRef] [PubMed]

76. Shoukry, N.M.; Salem, M.L.; Teleb, W.K.; Abdel Daim, M.M.; Abdel-Rahman, M.A. Antinociceptive, antiinflammatory, and antipyretic effects induced by the venom of Egyptian scorpion Androctonus amoreuxi. J. Basic Appl. Zool. 2020, 81, 56. [CrossRef]

77. Tran, T.V.; Hoang, A.N.; Nguyen, T.T.T.; Phung, T.V.; Nguyen, K.C.; Osipov, A.V.; Ivanov, I.A.; Tsetlin, V.I.; Utkin, Y.N. Anticoagulant activity of low-molecular weight compounds from Heterometrus laoticus scorpion venom. Toxins 2017, 9, 343. [CrossRef] [PubMed]

78. Yan, Y.Q.; Xie, J.; Wang, J.F.; Shi, Z.F.; Zhang, X.; Du, Y.P.; Zhao, X.C. Scorpion inhibits epithelial-mesenchymal transition and metastasis of hepatocellular carcinoma. Exp. Biol. Med. (Maywood) 2018, 243, 645-654. [CrossRef]

79. Kwon, K.B.; Kim, E.K.; Lim, J.G.; Jeong, E.S.; Shin, B.C.; Jeon, Y.S.; Kim, K.S.; Seo, E.A.; Ryu, D.G. Molecular mechanisms of apoptosis induced by Scorpio water extract in human hepatoma HepG2 cells. World J. Gastroenterol. 2005, 11, 943-947. [CrossRef] 
80. Al-Asmari, A.K.; Riyasdeen, A.; Islam, M. Scorpion Venom Causes Upregulation of p53 and Downregulation of Bcl-x(L) and BID Protein Expression by Modulating Signaling Proteins Erk(1/2) and STAT3, and DNA Damage in Breast and Colorectal Cancer Cell Lines. Integr. Cancer Ther. 2018, 17, 271-281. [CrossRef]

81. Wang, Y.; Li, K.; Han, S.; Tian, Y.H.; Hu, P.C.; Xu, X.L.; He, Y.Q.; Pan, W.T.; Gao, Y.; Zhang, Z.; et al. Chlorotoxin targets ER $\alpha /$ VASP signaling pathway to combat breast cancer. Cancer Med. 2019, 8, 1679-1693. [CrossRef] [PubMed]

82. Lazarou, J.; Pomeranz, B.H.; Corey, P.N. Incidence of adverse drug reactions in hospitalized patients: A meta-analysis of prospective studies. JAMA 1998, 279, 1200-1205. [CrossRef] [PubMed]

83. Coleman, J.J.; Pontefract, S.K. Adverse drug reactions. Clin. Med. (Lond.) 2016, 16, 481-485. [CrossRef] [PubMed]

84. Zeng, L.; Tang, G.; Wang, J.; Zhong, J.; Xia, Z.; Li, J.; Chen, G.; Zhang, Y.; Luo, S.; Huang, G.; et al. Safety and efficacy of herbal medicine for acute intracerebral hemorrhage (CRRICH): A multicentre randomised controlled trial. BMJ Open 2019, 9, e024932. [CrossRef]

85. Yuen, M.F.; Tam, S.; Fung, J.; Wong, D.K.; Wong, B.C.; Lai, C.L. Traditional Chinese medicine causing hepatotoxicity in patients with chronic hepatitis B infection: A 1-year prospective study. Aliment. Pharmacol. Ther. 2006, 24, 1179-1186. [CrossRef] [PubMed]

86. Wei, J.A.; Sun, L.M.; Chen, Y.X. Effects of Ailing Granule on immuno-reconstruction in HIV/AIDS patients. Chin. J. Integr. Tradit. West. Med. 2006, 26, 319-321.

87. Johnson, N.P.; Hummelshoj, L.; Adamson, G.D.; Keckstein, J.; Taylor, H.S.; Abrao, M.S.; Bush, D.; Kiesel, L.; Tamimi, R.; SharpeTimms, K.L.; et al. World Endometriosis Society consensus on the classification of endometriosis. Hum. Reprod. 2017, 32, 315-324. [CrossRef] [PubMed] 